FE622

\title{
Profile and Economic Impacts of Agriculture and Natural Resource Industries in the Suwannee River Basin Counties of Florida
}

\author{
M. Rahmani, A.W. Hodges, and W.D. Mulkey \\ University of Florida/Institute of Food and Agricultural Sciences \\ Food and Resource Economics Department \\ P.O. Box 110240, Gainesville, Fl 32611-0240 \\ Ph. (352)392-1881 X 315 or 312, Fax (352)392-3646 \\ Email: rahmani@ufl.edu or awhodges@ufl.edu
}

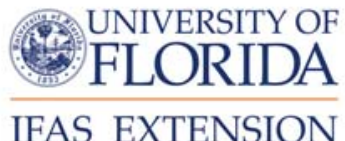

The Institute of Food and Agricultural Sciences (IFAS) is an Equal Opportunity Institution authorized to provide research, educational information and other services only to individuals and institutions that function with non-discrimination with respect to race, creed, color, religion, age, disability, sex, sexual orientation, marital status, national origin, political opinions or affiliations. U.S. Department of Agriculture, Cooperative Extension Service, University of Florida, IFAS, Florida A\&M University Cooperative Extension Program, and Boards of County Commissioners Cooperating. Larry Arrington, Dean 


\section{Executive Summary}

This report provides a profile of agricultural and natural resource industries and an overview of their economic impacts for a 14-county region in the Suwannee River Basin in Florida based on data from the IMPLAN Pro database for Florida counties for 2002 and data from the 2002 Census of Agriculture. Descriptive data include farm characteristics (number, distribution, farm land use, and investment in land and buildings); value of agricultural products; and measures of economic impacts such as output, value-added, and employment. Where possible, changes during the 1997-2002 period are highlighted. Additional reports are available for other major regions of Florida.

In 2002, the Suwannee River Basin counties of Florida had more than 7,000 farms and close to 200,000 acres of land used for various crops in the region. Livestock, poultry, and their products (4,274 farms) dominated the farm numbers in the Suwannee River Basin region. Agricultural lands in the Suwannee River Basin region were used mainly for cropland, nursery, greenhouse, floriculture, pasture, rangeland, and woodland. Forage (including all hays) was grown on more than 96,000 acres. The value of agricultural products sold in the Suwannee River Basin region exceeded $\$ 549$ million in 2002. The value of all livestock, poultry and their products exceeded $\$ 387$ million, accounting for 69 percent of the total value of all agricultural products for the region. Major components of the value of livestock, poultry, and their products included poultry and eggs (over $\$ 149$ million), milk cows and dairy products (\$134 million), and cattle and calves (\$45 million). The value of nursery, greenhouse, floriculture, and sod exceeded $\$ 47$ million, while the value of vegetables, melons, potatoes and sweet potatoes sold reached $\$ 42$ million.

The total economic impacts for all agriculture and natural resource industries in the Suwannee River Basin region exceeded $\$ 3.9$ billion in output in 2002. The forestry, wood, and paper products industy ( $\$ 1.7$ billion) ranked first in output impacts in the Suwannee River Basin region. Over 30,000 full- and part-time jobs were generated by agricultural and natural resource industries in the region. Forestry, wood, and paper products (the dominant industry group), produced more than 9,000 jobs and accounted for over 31 percent of total employment impacts for all industry groups in the region. The total value-added impacts of agricultural and natural resource industries exceeded $\$ 1.5$ billion in 2002. In terms of economic impacts, Taylor County ranked first in output impacts, and value-added impacts, while Suwannee County ranked first in employment impacts in the Suwannee River Basin region. 


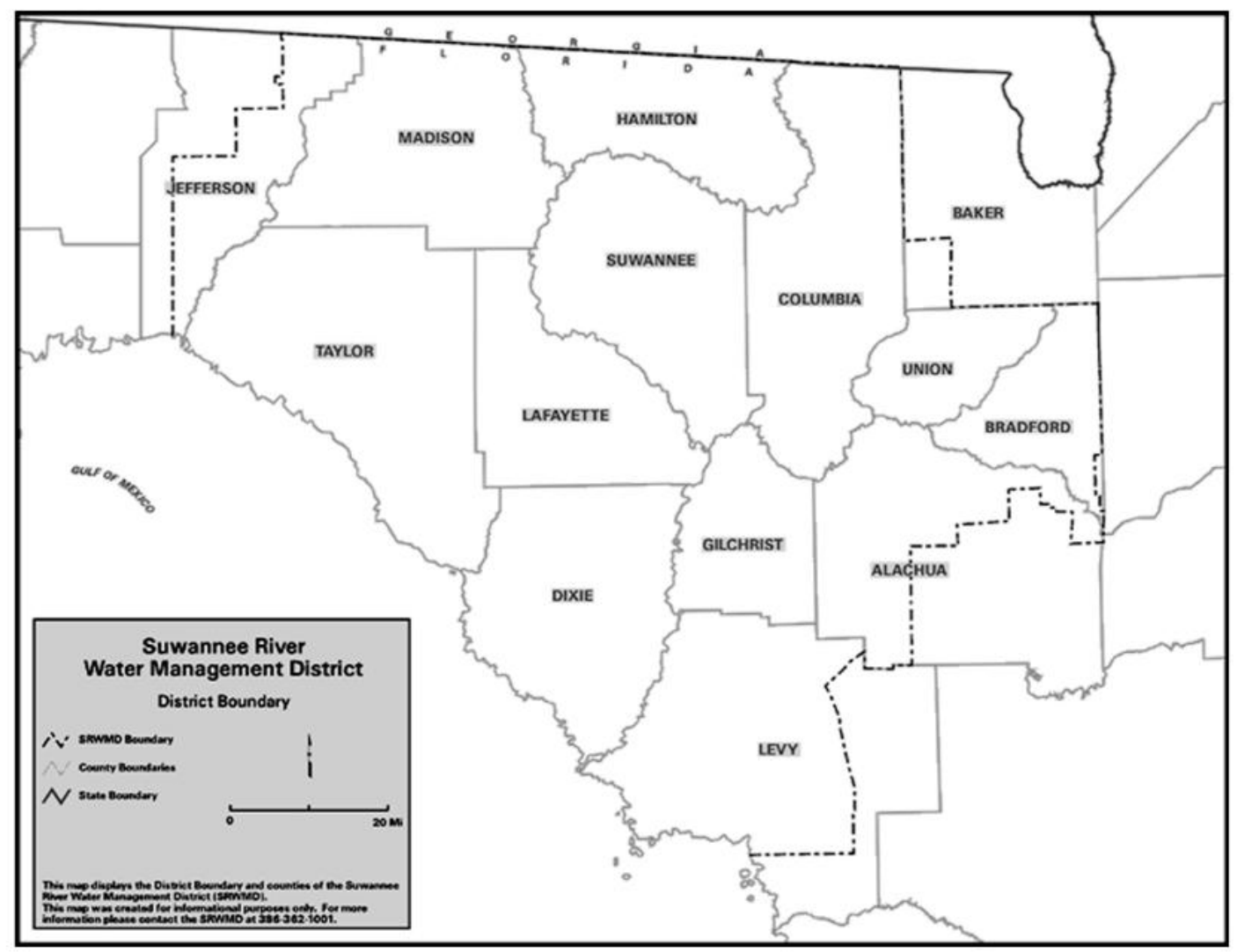

Source: Suwannee River Water Management District, February 26, 2002.

Figure 1. Suwannee River Water Management District map 


\section{Introduction}

This paper presents an analysis of the regional economic impacts of agriculture and natural resource industries in the Suwannee River Water Management District in north-central Florida. This region includes 14 counties, of which 10 are wholly inside district boundaries (Bradford, Columbia, Dixie, Gilchrist, Hamilton, Lafayette, Madison, Suwannee, Taylor, and Union), and 4 are partially within the district (Alachua, Baker, Levy, and Jefferson), as shown in Figure 1. The report provides a profile of agriculture and natural resource industries and an overview of their economic impacts for the Suwannee River Basin region. The predominant agricultural industries in this region include poultry, dairy, greenhouse/nursery, vegetables, and forestry. Food and fiber product manufacturing also contributes substantially to the region's economy, as do the related input supply and services sectors. The report is based on data from the IMPLAN Pro database for Florida counties for 2002 and data from the 2002 Census of Agriculture. ${ }^{1}$

Data from the 2002 Census of Agriculture $^{2}$ were used to augment the economic impact analysis and to allow for a detailed description of agricultural and natural resource industries for counties in each region. The 2002 Census of Agriculture, conducted by the National Agricultural Statistics Service of the U.S. Department of Agriculture, provides information at the county level on farm numbers; land use; market values of land, buildings, and machinery; crop and livestock sales; farm expenses; and income from federal programs. The data were reviewed by UF/IFAS county extension staff in 2004 in order to update as necessary.

\section{Agricultural Characteristics of the Region}

Data for this analysis were obtained from the 2002 USDA Census of Agriculture, and the Florida Agricultural Statistics Services. Information from the Census of Agriculture is summarized in Table 1. When data were available from more than one source, the most current source was used. Information was not adjusted for the 4 counties that lay partly outside the Suwannee River Basin.

\section{Farm Numbers and Farmland Use}

There were more than 7,000 farms in the Suwannee River Basin in 2002. Alachua County with 1,493 farms had the highest number of farms, followed by Suwannee County with 1,054 farms, Levy County with 897 farms, and Columbia County with 688 farms. Livestock, poultry, and their products ranked first (4,274 farms, or 60 percent of the regional total) and forage crops and all hays (1,440 farms) ranked second in the number of farms. The Suwannee River Basin had 127 tobacco farms and only 10 cotton farms. Farm numbers noted are based on the definition of a farm used by the 2002 Census of Agriculture: "any place from which $\$ 1,000$ or more of agricultural products were produced and sold, or normally would have been sold, during the census year." This definition was first used for the 1974 Census of Agriculture and has remained consistent since so that data are comparable across time periods.

\footnotetext{
1 IMPLAN Professional, Social Accounting \& Impact Analysis Software, 2 ${ }^{\text {nd }}$ Edition, June 2000, Minnesota IMPLAN Group, Inc., 1725 Tower Drive West, Suite 140, Stillwater, Minnesota 55082, www.implan.com

2002 Census of Agriculture, Florida, State and County Data, Volume 1, Geographic Area Series, Part 9, AC-02-A-

9, Issued June 2004, National Agricultural Statistics Service, USDA, http://www.usda.gov/nass/
} 
Agricultural lands in the Suwannee River Basin were used mainly for cropland, nursery, greenhouse, floriculture, pasture, rangeland, and woodland, with about 200,000 acres of land used for various crops in the region. Forage (including all hays) was grown on more than 96,000 acres; peanuts for nuts were grown on 26,000 acres; vegetables were grown on 21,000 acres, and corn for grain was grown on more than 18,000 acres of land. Levy County had the highest acreage for corn for silage (5,300 acres) and for peanuts (over 15,000 acres, or 57 percent of the regional total). Suwannee County with 17,000 acres had the highest acreage in forage crops.

\section{The Value of Agricultural Products Sold}

According to the 2002 Census of Agriculture, "market value of agricultural products sold represents the gross market value before taxes and production expenses of all agricultural products sold or removed from the place in 2002 regardless of who received the payment." Market value is equivalent to total sales, including the value of any shares received by partners, landlords, contractors, or others associated with the operation, and the value of commodities placed in Commodity Credit Corporation (CCC) loans. The market value of agricultural products sold does not include payments received for participation in other federal farm programs nor income from farm-related sources such as custom-work, other agricultural services, or income from non-farm sources.

The value of agricultural products sold in the Suwannee River Basin exceeded $\$ 549$ million in 2002. The value of all livestock, poultry, and their products was more than $\$ 387$ million, accounting for 69 percent of the total value of all agricultural products for the region. Major components of the value of livestock, poultry and their products included poultry and eggs with more than $\$ 149$ million, milk cows and dairy products with $\$ 134$ million, and cattle and calves with $\$ 45$ million.

The value of nursery, greenhouse, floriculture, and sod was over $\$ 47$ million, while the value of vegetables, melons, potatoes and sweet potatoes sold reached $\$ 42$ million. Other crops and hay sold were valued at more than $\$ 24$ million. The value of fruits, tree nuts, and berries sold was over $\$ 9$ million. Agricultural products sold directly to consumers were valued at more than $\$ 1$ million, and certified organically produced commodities sold was valued at \$200,000.

The value of all agricultural products sold in Suwannee County was $\$ 136$ million (the highest in the region), followed by Levy County with more than $\$ 83$ million. The livestock, poultry, and their products industry was responsible for the relatively high value of all agricultural products sold in both counties. Alachua County with $\$ 38$ million, or 24 percent of the total for the region, registered the highest value for crops.

\section{Economic Impact Analysis}

The IMPLAN Pro Economic Impact and Social Accounting software package, licensed to the University of Florida by the Minnesota IMPLAN Group, Inc., was used to generate multipliers for output, employment, and value-added for each agricultural and natural resource sector of the regional economy to allow a more comprehensive assessment of impacts. In addition to direct sales of the industries in question, IMPLAN multipliers capture inter-industry purchases (indirect 
effects) and employee household spending (induced effects). Regional IMPLAN models were constructed for each county using the county-level database provided with the IMPLAN software. No attempt was made to adjust for particular local conditions, and the analysis focused only on economic impacts resulting from operating revenues and employment created by agricultural and natural resource industries. In general, economic impact analysis permits decision makers and their advisors to look beyond the direct impacts or revenues generated by a business, industry, or event when considering its relationship and importance to a regional economy. Output, value-added, and employment are basic units for measuring economic activity, and permit comparison of one industry or event to another, and to the overall economy.

The IMPLAN software package and associated databases allow the estimation of multipliers to capture the total economic impact of agricultural and natural resource industries within a regional or local economy. This section reports the results of impact estimates for counties in the Suwannee River Basin for each of the agricultural sectors within IMPLAN. As noted above, estimates are based only on the IMPLAN data set with no attempt to adjust for particular local situations and the focus is only on impacts of sales and expenditures associated with farm operations and related manufacturing. ${ }^{3}$

For each reported measure (output, value-added and employment) for each county, the impacts reported include the direct output of the agricultural sector in question, the indirect impacts associated with inter-industry purchases of inputs by agriculture and its supplying industries, and the induced impacts of spending by employees and owners of agricultural and related industries. Reported impacts include activities within production agriculture, natural resource industry (forestry, mining, and fishing), food and forest products manufacturing, agricultural input supply, and agricultural services. Impacts are also reported in terms of labor income and indirect business taxes.

\section{Output Impacts}

Table 2 presents estimates of total revenue for agriculture and natural resource industries by sector and by county in the Suwannee River Basin. Total output for all counties in the region exceeded $\$ 2.8$ billion. Forestry and related wood and paper products was the largest industry, contributing $\$ 1.25$ billion in total output and accounting for 44 percent of the total output in the region. The livestock and dairy farming and animal products industry with more than $\$ 810$ million ranked second in the region. Agricultural inputs and services with \$394 million and the fruit and vegetable farming and processing industry with $\$ 128$ million were also major contributors to total output of agriculture and natural resource industries in the region.

There were some sectors within each industry group with notable output. Pulp mills (\$609 million), logging (\$235 million), sawmills (\$189 million), and forest nurseries and forest products and timber ( $\$ 98$ million) were the major sectors, accounting for more than 90 percent of output in the forestry, wood, and paper products industry in the region. Hamilton County with \$288 million in phosphatic fertilizer manufacturing accounted for 73 percent of the total output

\footnotetext{
${ }^{3}$ Readers desiring more information on IMPLAN should consult W.D. Mulkey \& A.W. Hodges, "Using Implan to Assess Local Economic Impacts," Food and Resource Economics Department, UF/IFAS, 2001, http://edis.ifas.ufl.edu/FE168.
} 
for agricultural inputs and services in the region. Poultry processing ( $\$ 287$ million), cattle ranching and farming (\$180 million), and poultry and egg production (\$130 million) were major sectors in the livestock and dairy farming and animal products industry.

In terms of output value, Taylor County with more than $\$ 739$ million ranked first. The forestry and related wood and paper product industry with $\$ 692$ million in Taylor County accounted for 56 percent of the direct output for this sector in the region and 94 percent of the direct output for the county. Suwannee County with $\$ 551$ million had the second largest output in the region. Almost 72 percent of the output impact in Suwannee County came from livestock and dairy farming and animal products. Phosphatic fertilizer manufacturing with \$315 million accounted for 91 percent of the output impact in Hamilton County.

Table 3 presents estimates of total output impacts for agriculture and natural resource industries by sector and by county for the Suwannee River Basin. Note again that this and the remaining impacts represent indirect and induced effects as well as direct effects. The total output impacts for all counties in the region exceeded $\$ 3.9$ billion. Forestry and related wood and paper products was the largest industry, contributing more than $\$ 1.7$ billion in total output impacts and accounting for more than one-third of the total in the region. The livestock and dairy farming and animal products industry with $\$ 1.2$ billion in output impacts ranked second in the region. Agricultural inputs and services with a total output impact of more than $\$ 516$ million was another major contributor to the total output impacts in the region.

Taylor County with more than $\$ 941$ million had the highest output impacts in the region, mainly in forestry and wood and paper products, particularly pulp mills, which produced $\$ 791$ million in output impacts. Suwannee County with $\$ 803$ million in livestock and dairy farming and animal products had the second highest total output impacts in the region. Hamilton County with $\$ 410$ million in output impacts ranked first in phosphatic fertilizer manufacturing in the region.

\section{Employment Impacts}

Employment impacts of agriculture and natural resource industries by county and industry group are reported in Table 4. More than 30,000 full- and part-time jobs were generated by agricultural and natural resource industries in the Suwannee River Basin. The forestry and wood and paper products manufacturing industry and the livestock and dairy farming and animal products industry (the dominant industry groups) each with 9,600 jobs produced most of the jobs (64 percent) in the region. Environmental horticulture (greenhouse/nursery production and landscape services) supported 2,900 jobs in the region (Figure2).

In terms of employment impacts, Suwannee County with more than 5,700 jobs in livestock and dairy farming and animal products dominated the employment outputs in the region. Alachua County with 5,200 jobs ranked second, and Taylor County with 4,200 jobs ranked third in the region. Environmental horticulture created most of the jobs in Alachua County, while forestry and wood and paper products manufacturing created most of the jobs in Taylor County. 


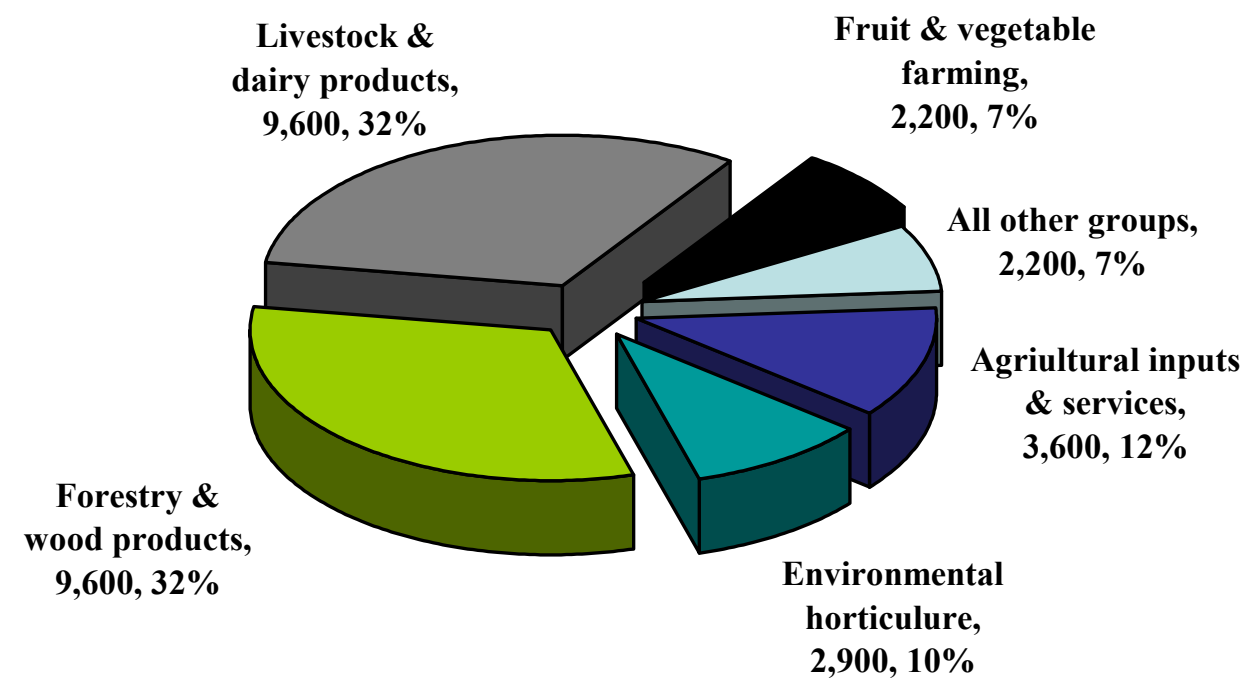

Figure 2. Employment impacts by industry group and number of jobs in Suwannee River Basin in Florida

\section{Value-added Impacts}

Value-added is the market value of a firm's output less the value of the inputs which it has purchased from others. It can be viewed as a net economic contribution by the industry sector after direct costs have been subtracted from gross sales. Value-added is the difference between the value of goods as they leave a particular stage of production and the costs of the goods as they entered that stage. Value-added includes payments made by industries to workers, interest, profits, and indirect business taxes. Total value-added impacts of agriculture and natural resource industries in the Suwannee River Basin exceeded $\$ 1.35$ billion (Table 5). Forestry, wood, and paper products with $\$ 570$ million accounted for 42 percent of the total value-added impacts for the region. Livestock and dairy farming and animal products with $\$ 295$ million and agricultural inputs and services with more than $\$ 175$ million were also major contributors to total valueadded impacts in the region (Figure 3).

Suwannee and Taylor Counties generated \$289 million and \$276 million in value-added impacts, respectively, and together accounted for 42 percent of the total value-added impacts in the Suwannee River Basin. Alachua County exceeded \$175 million in value-added impacts, and Levy and Hamilton Counties each had more than $\$ 100$ million in value-added impacts. 


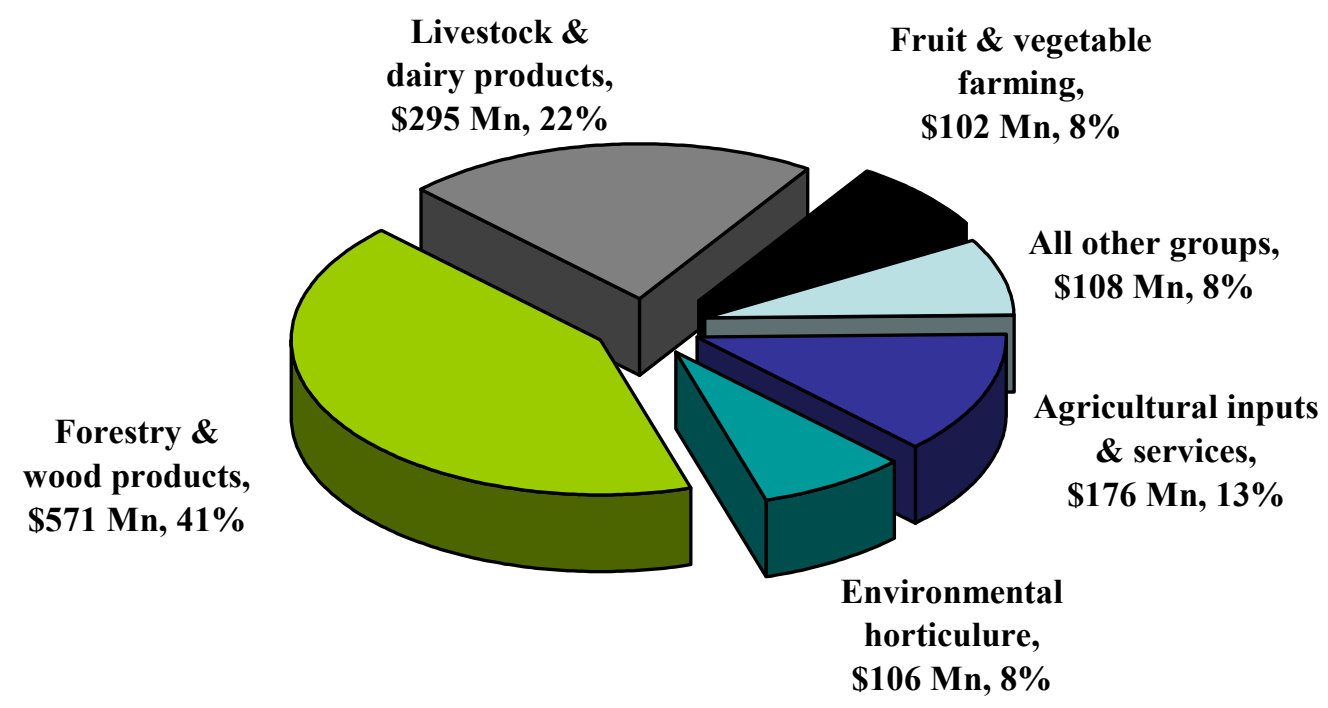

Figure 3. Value-added impacts by industry group in Suwannee River Basin of Florida

\section{Labor Income Impacts}

As a component of value-added, employee compensation includes wages, salary, health and life insurance, retirement payments, and any other non-cash compensation. Labor income impacts totaled more than $\$ 876$ million for the region (Tables 6). More than 42 percent of the total labor income impact was due to the activities in forestry, wood and paper production ( $\$ 373$ million). Other major contributors included the livestock and dairy farming and animal products industry with $\$ 172$ million, and agricultural input and services with $\$ 155$ million. For labor income impacts, Taylor County was the largest with more than \$ 199 million, followed by Suwannee County with $\$ 159$ million and Alachua County with $\$ 120$ million.

\section{Indirect Business Tax Impacts}

As another component of value-added, indirect business taxes consist of excise taxes, property taxes, fees, licenses, and sales taxes paid by businesses. These taxes occur during the normal operation of businesses but do not include taxes on profit or income. Indirect business tax impacts totaled more than $\$ 77$ million for the region (Table 7). Once again the forestry, wood and paper products industry with $\$ 31$ million and the livestock and dairy farming and animal products industry with $\$ 21$ million ranked highest in indirect business tax impacts in the region. Taylor County with $\$ 18$ million ranked first, Suwannee County with $\$ 16$ million ranked second, and Alachua County with more than $\$ 10$ million ranked third in indirect business tax impacts.

\section{Acknowledgement}

Constructive comments given by reviewer, Dr. John J. Haydu, are greatly appreciated. 
Table 1. Summary of agricultural commodity volumes produced, number of farms, and acreage in the Suwannee River Basin counties of Florida, 2002

\begin{tabular}{|c|c|c|c|c|c|c|c|c|c|c|c|c|c|c|c|c|}
\hline Item & 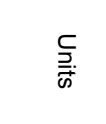 & 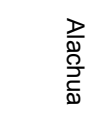 & 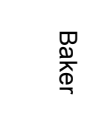 & 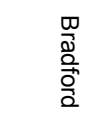 & 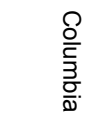 & $\frac{\bar{y}}{\mathrm{x}}$ & 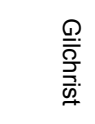 & 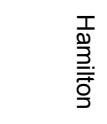 & 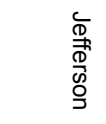 & 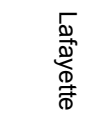 & $\stackrel{\infty}{<}$ & $\begin{array}{l}\frac{3}{30} \\
\frac{0}{0.0} \\
\stackrel{0}{3}\end{array}$ & 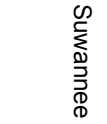 & 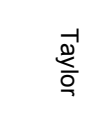 & $\begin{array}{l}\text { ㄷ․ㅁ. } \\
\text { o. }\end{array}$ & $\begin{array}{l}\text { Total All } \\
\text { Counties }\end{array}$ \\
\hline Farms & number & 1,493 & 204 & 378 & 688 & 215 & 408 & 239 & 418 & 195 & 897 & 529 & 1,054 & 101 & 275 & 7,094 \\
\hline Value of all ag products sold & $\$ 1000$ & 58,665 & 25,425 & 17,913 & 46,767 & 6,542 & 44,558 & 12,169 & 21,154 & 48,116 & 83,226 & 24,645 & 135,960 & 13,354 & 10,711 & 549,205 \\
\hline Value of crops & $\$ 1000$ & 37,620 & 9,515 & 1,673 & 7,947 & (D) & 6,194 & 6,440 & 9,317 & 3,355 & 25,949 & 10,068 & 32,497 & 727 & 4,275 & 155,577 \\
\hline $\begin{array}{l}\text { Value of livestock, poultry \& } \\
\text { products }\end{array}$ & $\$ 1000$ & 21,044 & 15,911 & 16,240 & 38,820 & (D) & 38,364 & 5,728 & 11,837 & 44,762 & 57,277 & 14,577 & 103,464 & 12,627 & 6,436 & 387,087 \\
\hline \multirow{2}{*}{$\begin{array}{l}\text { Grains, oilseeds, dry beans } \\
\& \text { dry peas }\end{array}$} & \# farms & 31 & 4 & 1 & 7 & 2 & 9 & 30 & 20 & 8 & 12 & 46 & 34 & - & 2 & 206 \\
\hline & $\$ 1000$ & (D) & 7 & (D) & (D) & (D) & 572 & 4,500 & 1,636 & (D) & (D) & 1,439 & 1,490 & - & (D) & 9,644 \\
\hline \multirow[t]{3}{*}{ Corn for grain } & \# farms & 22 & 10 & 4 & 7 & - & 8 & 54 & 26 & 2 & 15 & 64 & 30 & - & 7 & 249 \\
\hline & Acres & 777 & 38 & 79 & 137 & - & 219 & 5,700 & 4,091 & (D) & 483 & 4,761 & 1,807 & - & 60 & 18,152 \\
\hline & Bushels & 23,342 & 2,680 & 4,790 & 4,747 & - & 8,675 & 294,533 & 543,685 & (D) & 18,113 & 317,962 & 178,316 & - & 4,080 & $1,400,923$ \\
\hline \multirow[t]{3}{*}{ Corn for silage or greenchop } & \# farms & 25 & - & - & 2 & 1 & 9 & - & 3 & 7 & 12 & - & 20 & - & - & 79 \\
\hline & Acres & 4,677 & - & - & (D) & (D) & 2,327 & - & 1,218 & 847 & 5,326 & - & 3,528 & - & - & 17,923 \\
\hline & Bushels & 79,674 & - & - & (D) & (D) & 29,683 & - & 20,167 & 18,213 & 91,004 & - & 64,794 & - & - & 303,535 \\
\hline \multirow[t]{2}{*}{ Wheat for grain } & \# farms & - & - & - & - & - & 1 & 1 & - & - & 1 & - & - & - & - & 3 \\
\hline & Bushels & - & - & - & - & - & (D) & (D) & - & - & (D) & - & - & - & - & 0 \\
\hline Oats for grain & \# farms & - & - & - & 2 & - & - & 1 & 1 & - & 2 & 1 & 2 & - & - & 9 \\
\hline \multirow[t]{2}{*}{ Sorghum for grain } & \# farms & - & - & - & - & - & 1 & - & 1 & - & - & 1 & - & - & - & 3 \\
\hline & Acres & - & - & - & - & - & (D) & - & (D) & - & - & (D) & - & - & - & 0 \\
\hline \multirow[t]{3}{*}{ Sorghum for silage or greenchop } & \# farms & 5 & - & - & 1 & 1 & 7 & - & 2 & 1 & 3 & - & 4 & - & - & 24 \\
\hline & Acres & 625 & - & - & (D) & (D) & 1,164 & - & (D) & (D) & (D) & - & 205 & - & - & 1,994 \\
\hline & Tons & 6,400 & - & - & (D) & (D) & 10,500 & - & (D) & (D) & (D) & - & 2,180 & - & - & 19,080 \\
\hline \multirow[t]{3}{*}{ Soybeans for beans } & \# farms & - & - & - & 1 & - & - & 2 & 5 & - & - & 9 & 2 & - & - & 19 \\
\hline & Acres & - & - & - & (D) & - & - & (D) & (D) & - & - & 1,946 & (D) & - & - & 1,946 \\
\hline & Bushels & - & - & - & (D) & - & - & (D) & (D) & - & - & 78,867 & (D) & - & - & 78,867 \\
\hline
\end{tabular}


Table 1. Summary of agricultural commodity volumes produced, number of farms, and acreage in the Suwannee River Basin counties of Florida, 2002

\begin{tabular}{|c|c|c|c|c|c|c|c|c|c|c|c|c|c|c|c|c|}
\hline Item & $\underset{\mathrm{c}}{\mathrm{c}}$ & 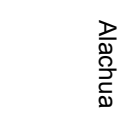 & 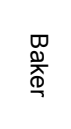 & $\begin{array}{l}\frac{w}{0} \\
\stackrel{0}{0} \\
\frac{0}{0} \\
\frac{0}{\alpha}\end{array}$ & $\frac{ᄋ}{\frac{\varrho}{\frac{O}{5}}}$ & $\begin{array}{l}\text { D. } \\
\stackrel{x}{\bar{D}}\end{array}$ & 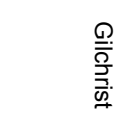 & 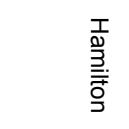 & 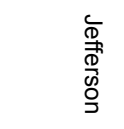 & 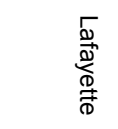 & $\stackrel{\text { D }}{<}$ & $\begin{array}{l}3 \\
\frac{3}{0} \\
\frac{2}{0 .} \\
\stackrel{0}{3}\end{array}$ & $\begin{array}{l}\infty \\
\sum_{\infty}^{\infty} \\
\sum_{\overparen{D}} \\
\mathbb{D} \\
\mathbb{D}\end{array}$ & 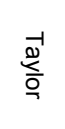 & ডั. & $\begin{array}{l}\text { Total All } \\
\text { Counties }\end{array}$ \\
\hline \multirow[t]{4}{*}{ Cotton \& cottonseed } & \# farms & - & - & - & 1 & - & - & 2 & 5 & - & - & - & 2 & - & - & 10 \\
\hline & Acres & - & - & - & (D) & - & - & (D) & 1,288 & - & - & - & (D) & - & - & 1,288 \\
\hline & Bales & - & - & - & (D) & - & - & (D) & 1,298 & - & - & - & (D) & - & - & 1,298 \\
\hline & $\$ 1000$ & - & - & - & (D) & - & - & (D) & 193 & - & - & - & (D) & - & - & 193 \\
\hline \multirow[t]{4}{*}{ Tobacco } & \# farms & 20 & 1 & 2 & 14 & - & 1 & 27 & 2 & 8 & 2 & 14 & 22 & - & 14 & 127 \\
\hline & Acres & 637 & (D) & 49 & 459 & - & (D) & 645 & (D) & 139 & (D) & 576 & 1,175 & - & 154 & 3,834 \\
\hline & Pounds & $1,484,600$ & (D) & - & 50,990 & - & (D) & $1,240,560$ & (D) & 419,175 & (D) & $1,291,256$ & $3,087,210$ & - & $\begin{array}{r}217,15 \\
3\end{array}$ & $8,990,944$ \\
\hline & $\$ 1000$ & 1,861 & (D) & - & 2,202 & - & (D) & 2,226 & (D) & 801 & (D) & 1,993 & 5,350 & - & 464 & 14,897 \\
\hline \multirow[t]{3}{*}{ Potatoes } & \# farms & 3 & - & - & 1 & - & - & - & 5 & - & 2 & - & 1 & - & 1 & 13 \\
\hline & Acres & (Z) & - & - & (D) & - & - & - & 3 & - & (D) & - & (D) & - & (D) & 3 \\
\hline & CWT & 40 & - & - & (D) & - & - & - & 500 & - & (D) & - & (D) & - & (D) & 540 \\
\hline Sweet potatoes & \# farms & 1 & - & - & - & - & - & 2 & - & 2 & - & 2 & 2 & - & - & 9 \\
\hline \multirow[t]{3}{*}{ Peanuts for nuts } & \# farms & 30 & - & - & 5 & - & 9 & 2 & 11 & 3 & 27 & 11 & 19 & - & 6 & 123 \\
\hline & Acres & 1,782 & - & - & (D) & - & 2,000 & (D) & 1,494 & 9 & 15,296 & 1,757 & 4,218 & - & 30 & 26,586 \\
\hline & Pounds & $5,832,968$ & - & - & (D) & & $3,800,000$ & (D) & $5,046,008$ & (D) & $50,666,166$ & $5,213,121$ & $9,456,151$ & - & 73,500 & $80,087,914$ \\
\hline Rice & \# farms & - & - & 1 & - & - & - & - & - & - & - & - & - & - & - & 1 \\
\hline \multirow[t]{2}{*}{ Vegetables harvested for sale } & \# farms & 113 & 17 & 28 & 23 & 4 & 10 & 20 & 29 & 7 & 42 & 21 & 36 & - & 11 & 361 \\
\hline & Acres & 5,887 & 225 & 550 & 199 & (D) & 854 & 2,500 & 256 & 241 & 2,251 & 519 & 6,945 & - & 1,444 & 21,871 \\
\hline \multirow{2}{*}{$\begin{array}{l}\text { Vegetables, melons, potatoes \& } \\
\text { sweet potatoes }\end{array}$} & \# farms & 111 & 17 & 28 & 23 & 4 & 10 & 8 & 34 & 7 & 42 & 21 & 37 & - & 11 & 353 \\
\hline & $\$ 1000$ & 12,859 & (D) & 634 & 437 & (D) & 1,723 & 2,066 & 331 & 453 & 4,387 & 1,273 & 16,216 & - & 1,555 & 41,934 \\
\hline \multirow[t]{2}{*}{ Orchards } & \# farms & 156 & 14 & 24 & 61 & 4 & 25 & 11 & 81 & 9 & 23 & 37 & 66 & 1 & 24 & 536 \\
\hline & Acres & 2,179 & 129 & 229 & 325 & 34 & 176 & 101 & 1,101 & 63 & 125 & 410 & 732 & (D) & 289 & 5,893 \\
\hline \multirow[t]{2}{*}{ Fruits, tree nuts \& berries } & \# farms & 141 & 10 & 19 & 38 & 6 & 22 & 3 & 57 & 6 & 23 & 26 & 47 & 3 & 22 & 423 \\
\hline & $\$ 1000$ & 8,113 & 40 & (D) & 202 & 11 & (D) & (D) & 888 & 53 & 185 & (D) & (D) & (D) & (D) & 9,492 \\
\hline
\end{tabular}


Table 1. Summary of agricultural commodity volumes produced, number of farms, and acreage in the Suwannee River Basin counties of Florida, 2002

\begin{tabular}{|c|c|c|c|c|c|c|c|c|c|c|c|c|c|c|c|c|}
\hline Item & $\stackrel{\subsetneq}{\stackrel{F}{F}}$ & 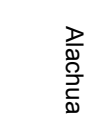 & 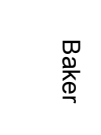 & 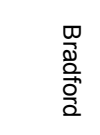 & $\begin{array}{l}\frac{\bigcirc}{\frac{\rho}{5}} \\
\frac{\bar{g}}{\Phi}\end{array}$ & $\frac{\bar{x}}{\mathrm{x}}$ & 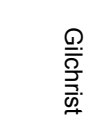 & 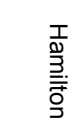 & 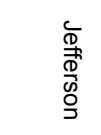 & 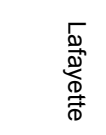 & $\stackrel{\text { D }}{<}$ & $\begin{array}{l}3 \\
\frac{3}{0} \\
\frac{2}{0 .} \\
0 \\
0\end{array}$ & 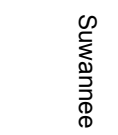 & $\stackrel{\vec{\aleph}}{\stackrel{\aleph}{ }}$ & ড়. & $\begin{array}{l}\text { Total All } \\
\text { Counties }\end{array}$ \\
\hline \multirow{2}{*}{$\begin{array}{l}\text { Nursery, greenhouse, floriculture } \\
\& \text { sod }\end{array}$} & \# farms & 128 & 12 & 11 & 30 & 4 & 16 & 12 & 33 & 4 & 25 & 15 & 27 & 3 & 4 & 324 \\
\hline & $\$ 1000$ & 11,122 & 8,780 & 399 & (D) & (D) & 2,127 & 607 & 5,086 & (D) & 10,676 & 2,955 & 4,307 & (D) & 1,448 & 47,507 \\
\hline \multirow{2}{*}{$\begin{array}{l}\text { Cut Christmas trees \& } \\
\text { short-rotation woody crops }\end{array}$} & \# farms & 4 & 1 & 2 & - & - & 3 & - & 3 & - & - & 2 & 3 & 2 & - & 20 \\
\hline & $\$ 1000$ & (D) & (D) & (D) & - & - & 16 & - & (D) & - & - & (D) & (D) & (D) & - & 16 \\
\hline \multirow[t]{3}{*}{ Forage (all hay and all haylage) } & \# farms & 278 & 24 & 95 & 112 & 15 & 104 & 58 & 43 & 48 & 140 & 141 & 291 & 18 & 73 & 1,440 \\
\hline & Acres & 22,371 & 570 & 5,200 & 5,558 & 1,683 & 8,504 & 2,437 & 2,881 & 4,976 & 12,416 & 8,098 & 17,562 & 795 & 3,555 & 96,606 \\
\hline & Dry tons & 62,167 & 2,080 & 15,092 & 20,250 & 5,736 & 24,973 & 7,983 & 7,953 & 20,233 & 35,817 & 26,245 & 63,750 & 2,480 & 14,869 & 309,628 \\
\hline \multirow[t]{2}{*}{ Other crops \& hay } & \# farms & 152 & 6 & 39 & 50 & 3 & 64 & 24 & 34 & 18 & 87 & 61 & 165 & 6 & 40 & 749 \\
\hline & $\$ 1000$ & 2,748 & 31 & 514 & (D) & 99 & 1,530 & 466 & 998 & 620 & 10,397 & 1,423 & 4,070 & 79 & 459 & 23,434 \\
\hline \multirow[t]{2}{*}{ Livestock, poultry \& products } & \# farms & 808 & 135 & 244 & 418 & 180 & 223 & 131 & 174 & 126 & 616 & 317 & 641 & 70 & 193 & 4,276 \\
\hline & $\$ 1000$ & 21,044 & 15,911 & 16,240 & 38,820 & (D) & 38,364 & 5,728 & 11,837 & 44,762 & 57,277 & 14,577 & 103,464 & 12,627 & 6,436 & 387,087 \\
\hline \multirow[t]{4}{*}{ Cattle \& calves } & \# farms & 765 & 137 & 241 & 454 & 97 & 227 & 128 & 136 & 118 & 460 & 337 & 587 & 75 & 207 & 3,969 \\
\hline & Inventory & 48,998 & 4,256 & 11,103 & 19,324 & 5,218 & 25,690 & 7,928 & 12,525 & 20,173 & 48,691 & 22,049 & 41,253 & 4,376 & 8,919 & 280,503 \\
\hline & $\$ 1000$ & 9,402 & (D) & 3,417 & (D) & 861 & 5,063 & 1,182 & 2,126 & 2,521 & 7,965 & 3,796 & 6,299 & 846 & 1,544 & 45,022 \\
\hline & \# sold & 22,344 & 2,064 & 6,101 & 9,239 & 2,782 & 14,929 & 3,275 & 5,497 & 8,319 & 23,665 & 10,429 & 17,370 & 2,289 & 4,030 & 132,333 \\
\hline \multirow[t]{2}{*}{ Beef cows } & \# farms & 610 & 108 & 191 & 372 & 77 & 183 & 118 & 107 & 86 & 347 & 252 & 476 & 60 & 146 & 3,133 \\
\hline & Inventory & 24,090 & (D) & (D) & 11,800 & 2,331 & 6,820 & (D) & 4,702 & 3,812 & 18,168 & 12,671 & 16,859 & (D) & 4,101 & 105,354 \\
\hline \multirow[t]{3}{*}{ Milk cows \& dairy products } & \# farms & 42 & 4 & 5 & 19 & 9 & 14 & 3 & 4 & 23 & 15 & 11 & 45 & 2 & 13 & 209 \\
\hline & Inventory & 2,495 & (D) & (D) & 790 & 1,403 & 11,344 & (D) & 3,217 & 10,891 & 12,105 & 61 & 8,784 & (D) & 425 & 51,515 \\
\hline & $\$ 1000$ & 5,579 & (D) & (D) & 1,111 & 2,080 & 32,307 & (D) & 9,590 & 23,373 & 38,361 & 140 & 20,208 & - & 1,020 & 133,769 \\
\hline \multirow[t]{4}{*}{ Hogs \& pigs } & \# farms & 71 & 11 & 7 & 45 & 16 & 21 & 7 & 25 & 16 & 33 & 21 & 32 & 9 & 8 & 322 \\
\hline & Inventory & 1,439 & 455 & (D) & 664 & 689 & 151 & (D) & 431 & (D) & 1,078 & (D) & 478 & 95 & 296 & 5,776 \\
\hline & \# sold & 1,254 & 386 & (D) & 546 & 380 & 383 & 6,019 & 246 & (D) & 1,329 & (D) & 976 & 68 & 799 & 12,386 \\
\hline & $\$ 1000$ & 78 & 13 & (D) & 27 & (D) & (D) & (D) & 21 & 117 & 52 & (D) & 55 & (D) & 37 & 400 \\
\hline
\end{tabular}


Table 1. Summary of agricultural commodity volumes produced, number of farms, and acreage in the Suwannee River Basin counties of Florida, 2002

\begin{tabular}{|c|c|c|c|c|c|c|c|c|c|c|c|c|c|c|c|c|}
\hline Item & $\stackrel{\subsetneq}{\stackrel{f}{\risingdotseq}}$ & $\begin{array}{l}\vec{D} \\
\stackrel{\vec{D}}{\underline{D}} \\
\overrightarrow{\underline{D}}\end{array}$ & 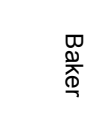 & 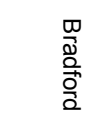 & 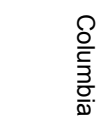 & $\frac{\mathrm{D}}{\mathrm{x}}$ & 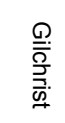 & 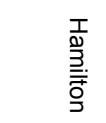 & 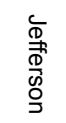 & 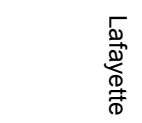 & $\stackrel{\text { Do }}{\leqslant}$ & $\begin{array}{l}3 \\
\frac{3}{0} \\
\frac{2}{0} \\
\stackrel{0}{9}\end{array}$ & 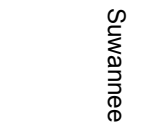 & 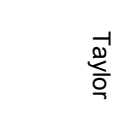 & ํํำ & $\begin{array}{l}\text { Total All } \\
\text { Counties }\end{array}$ \\
\hline \multirow[t]{3}{*}{ Sheep, goats \& products } & \# farms & 76 & 2 & 15 & 43 & 13 & 13 & 11 & 24 & 11 & 19 & 13 & 28 & 1 & 15 & 284 \\
\hline & $\$ 1000$ & 76 & (D) & 6 & 22 & 4 & 52 & 6 & 14 & 8 & 17 & 14 & 25 & (D) & 17 & 261 \\
\hline & Inventory & 756 & 4 & 42 & 181 & - & 180 & - & 532 & (D) & 105 & 26 & 4 & - & (D) & 1,830 \\
\hline \multirow[t]{2}{*}{ Poultry \& eggs } & \# farms & 41 & 26 & 22 & 16 & 10 & 3 & 16 & 21 & 33 & 13 & 20 & 111 & 12 & 11 & 355 \\
\hline & $\$ 1000$ & (D) & 13,392 & 10,921 & (D) & 1,130 & (D) & 3,471 & 9 & 18,708 & 2 & 9,998 & 76,278 & 11,731 & 3,770 & 149,410 \\
\hline \multirow{2}{*}{$\begin{array}{l}\text { Layer Chickens } 20 \text { weeks old } \\
\text { \& older }\end{array}$} & \# farms & 69 & 13 & 17 & 43 & 9 & 18 & 7 & 19 & 12 & 33 & 15 & 58 & 9 & 20 & 342 \\
\hline & Inventory & 1,320 & 305 & (D) & (D) & 78,334 & 242 & (D) & 544 & 89,600 & 430 & (D) & 322,268 & (D) & (D) & 493,043 \\
\hline \multirow{2}{*}{$\begin{array}{l}\text { Broilers \& other meat-type } \\
\text { chickens }\end{array}$} & \# farms & 7 & 0 & 0 & 7 & - & - & 5 & 2 & 26 & - & 11 & 83 & 10 & 3 & 154 \\
\hline & \# sold & (D) & 0 & 0 & 600,028 & - & - & $, 790,000$ & (D) & $10,770,270$ & - & $5,909,117$ & $32,874,136$ & $2,480,550$ & (D) & $54,424,101$ \\
\hline \multirow{2}{*}{$\begin{array}{l}\text { Horses, ponies, mules, burros } \\
\& \text { donkeys }\end{array}$} & \# farms & 149 & 20 & 32 & 40 & 3 & 42 & 4 & 34 & 8 & 146 & 23 & 73 & 8 & 13 & 595 \\
\hline & $\$ 1000$ & 878 & 97 & 204 & 65 & 17 & 538 & (D) & 70 & 28 & 2,814 & 43 & 289 & 28 & 31 & 5,102 \\
\hline \multirow[t]{2}{*}{ Aquaculture } & \# farms & 8 & - & 10 & 4 & 76 & - & - & - & 3 & 136 & 1 & 7 & - & 5 & 250 \\
\hline & $\$ 1000$ & 183 & - & (D) & 9 & 316 & - & - & - & (D) & 7,901 & (D) & 33 & - & $(Z)$ & 8,442 \\
\hline \multirow[t]{2}{*}{ Other animals \& products } & \# farms & 28 & 5 & - & 18 & 5 & 6 & 7 & 7 & 1 & 16 & 8 & 11 & 5 & 5 & 122 \\
\hline & $\$ 1000$ & (D) & (D) & - & 423 & 26 & 378 & 40 & 7 & (D) & 165 & (D) & 276 & (D) & 17 & 1,332 \\
\hline \multirow{2}{*}{$\begin{array}{l}\text { Ag products sold directly to } \\
\text { consumers }\end{array}$} & \# farms & 123 & 13 & 18 & 30 & 12 & 14 & 7 & 34 & 9 & 41 & 22 & 41 & 9 & 17 & 390 \\
\hline & $\$ 1000$ & 679 & 18 & 28 & 47 & 7 & 12 & 3 & 32 & 22 & 101 & 89 & 83 & 12 & 28 & 1,161 \\
\hline \multirow{2}{*}{$\begin{array}{l}\text { Certified organically produced } \\
\text { commodities }\end{array}$} & \# farms & 15 & 1 & 1 & 4 & 1 & - & - & 9 & - & 2 & 1 & 1 & - & 2 & 37 \\
\hline & $\$ 1000$ & 198 & (D) & (D) & 4 & (D) & - & - & (D) & - & (D) & (D) & (D) & - & (D) & 202 \\
\hline
\end{tabular}

(D): Nondisclosed due to small number of firms reporting 3,2004 , by the National Agricultural Statistics Service (NASS), Agricultural Statistics Board, U.S. Department of Agriculture.

Some information revised by UF/IFAS Extension. 
Table 2. Output (revenue) of agriculture and natural resource industries, Suwannee River Basin counties of Florida, 2002 data

\begin{tabular}{|c|c|c|c|c|c|c|c|c|c|c|c|c|c|c|c|}
\hline Industry Group & $\begin{array}{l}\frac{D}{\mathcal{D}} \\
\stackrel{\tilde{D}}{\vec{D}} \\
\stackrel{D}{D}\end{array}$ & 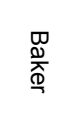 & $\begin{array}{l}\frac{w}{0} \\
\frac{0}{0} \\
\frac{0}{0} \\
\frac{0}{2}\end{array}$ & 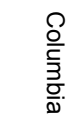 & 莃: & 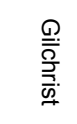 & 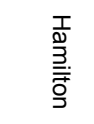 & 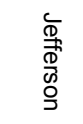 & 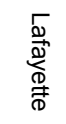 & $\stackrel{\text { Do }}{\xi}$ & $\begin{array}{l}3 \\
\frac{3}{0} \\
\frac{0}{0 .} \\
0 \\
0\end{array}$ & 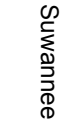 & $\stackrel{\stackrel{-1}{\stackrel{0}{O}}}{\underline{y}}$ & ํ. & $\begin{array}{l}\text { Grand } \\
\text { Total }\end{array}$ \\
\hline Agricultural Inputs \& Services & \multicolumn{14}{|c|}{ <>- } & \\
\hline Agriculture \& forestry activities & 12.2 & 0.0 & 0.2 & 2.7 & 0.0 & 1.3 & 0.3 & 1.2 & 0.1 & 17.0 & 9.4 & 3.5 & 3.5 & 0.2 & 51.5 \\
\hline Farm machinery \& equipment mfg & 0.0 & 0.0 & 0.0 & 0.0 & 0.0 & 0.0 & 0.0 & 0.0 & 0.0 & 0.0 & 0.0 & 1.0 & 0.0 & 0.0 & 1.0 \\
\hline Fertilizer, mixing only, mfg & 0.2 & 0.0 & 0.0 & 0.0 & 0.0 & 0.0 & 0.0 & 0.0 & 0.0 & 0.0 & 0.0 & 0.0 & 0.0 & 0.7 & 0.9 \\
\hline Food product machinery mfg & 0.0 & 0.0 & 0.0 & 0.0 & 0.0 & 0.0 & 0.0 & 0.4 & 0.0 & 0.0 & 0.0 & 0.0 & 0.0 & 0.0 & 0.4 \\
\hline Lawn/garden equipment mfg & 0.0 & 0.0 & 0.0 & 0.0 & 0.0 & 0.0 & 0.0 & 1.0 & 0.0 & 0.0 & 0.0 & 0.0 & 0.0 & 0.0 & 1.0 \\
\hline New farm housing units/additions & 7.8 & 0.5 & 0.6 & 1.8 & 1.3 & 0.3 & 0.2 & 0.4 & 0.0 & 1.2 & 0.2 & 0.8 & 0.7 & 0.2 & 15.7 \\
\hline Phosphatic fertilizer mfg & 0.0 & 0.0 & 0.0 & 0.0 & 0.0 & 0.0 & 288.1 & 0.0 & 0.0 & 0.0 & 0.0 & 0.0 & 0.0 & 0.0 & 288.1 \\
\hline Veterinary services & 21.6 & 1.4 & 0.8 & 2.2 & 0.3 & 0.2 & 0.7 & 1.6 & 0.2 & 2.7 & 0.3 & 2.9 & 0.6 & 0.0 & 35.3 \\
\hline Total & 41.7 & 1.8 & 1.5 & 6.7 & 1.6 & 1.8 & 289.4 & 4.5 & 0.3 & 20.9 & 9.8 & 8.2 & 4.8 & 1.1 & 394.1 \\
\hline \multicolumn{16}{|l|}{ Environmental Horticulture } \\
\hline Greenhouse/nursery production & 6.9 & 4.3 & 0.2 & 1.6 & 1.1 & 4.1 & 0.2 & 12.9 & 2.2 & 18.1 & 5.8 & 3.2 & 0.4 & 0.4 & 61.6 \\
\hline Landscape services ( $70 \%$ bldgs) & 35.7 & 0.6 & 2.6 & 5.5 & 0.2 & 0.5 & 0.3 & 1.4 & 0.1 & 1.9 & 1.0 & 2.8 & 1.8 & 1.0 & 55.4 \\
\hline Total & 42.6 & 4.9 & 2.8 & 7.1 & 1.3 & 4.7 & 0.6 & 14.3 & 2.3 & 20.0 & 6.9 & 6.0 & 2.2 & 1.4 & 117.1 \\
\hline \multicolumn{16}{|l|}{ Fishing \& Wildlife } \\
\hline Fishing & 0.0 & 0.0 & 0.0 & 0.0 & 0.0 & 0.0 & 0.0 & 0.0 & 0.0 & 1.9 & 0.0 & 0.0 & 0.0 & 0.0 & 1.9 \\
\hline Wildlife (hunting \& trapping) & 0.0 & 0.0 & 0.0 & 0.0 & 0.0 & 0.0 & 0.0 & 12.2 & 0.0 & 0.0 & 0.0 & 0.0 & 0.0 & 0.0 & 12.2 \\
\hline Total & 0.0 & 0.0 & 0.0 & 0.0 & 0.0 & 0.0 & 0.0 & 12.2 & 0.0 & 1.9 & 0.0 & 0.0 & 0.0 & 0.0 & 14.0 \\
\hline \multicolumn{16}{|l|}{ Forestry, Wood \& Paper Product Mfg } \\
\hline Cut stock/resawing lumber/planting & 0.0 & 0.0 & 0.0 & 15.5 & 0.0 & 0.0 & 0.0 & 0.0 & 0.0 & 0.0 & 0.0 & 0.0 & 0.0 & 0.0 & 15.5 \\
\hline Engineered wood \& truss mfg & 12.7 & 0.0 & 0.0 & 0.5 & 0.0 & 1.3 & 0.0 & 0.0 & 0.6 & 0.0 & 0.0 & 0.0 & 0.0 & 0.0 & 15.1 \\
\hline Envelope manufacturing & 7.6 & 0.0 & 0.0 & 0.0 & 0.0 & 0.0 & 0.0 & 0.0 & 0.0 & 0.0 & 0.0 & 0.0 & 0.0 & 0.0 & 7.6 \\
\hline Forest nurseries/products/timber & 20.5 & 6.6 & 0.0 & 0.0 & 2.5 & 7.7 & 2.2 & 3.3 & 1.0 & 1.6 & 2.4 & 38.4 & 11.6 & 0.0 & 97.8 \\
\hline Logging & 6.2 & 2.0 & 8.0 & 15.8 & 29.0 & 5.1 & 1.6 & 21.8 & 3.2 & 26.5 & 16.8 & 45.8 & 43.6 & 9.3 & 234.8 \\
\hline Miscellaneous wood product mfg & 0.0 & 0.0 & 0.4 & 0.3 & 0.0 & 1.6 & 0.0 & 0.0 & 0.0 & 0.0 & 0.0 & 0.3 & 0.0 & 0.0 & 2.6 \\
\hline Other millwork, including flooring & 4.8 & 0.0 & 0.0 & 0.2 & 0.0 & 0.0 & 0.0 & 0.0 & 0.0 & 0.0 & 0.0 & 0.0 & 0.0 & 0.0 & 5.0 \\
\hline Prefabricated wood building $\mathrm{mfg}$. & 0.1 & 0.0 & 0.0 & 0.0 & 0.0 & 0.0 & 0.0 & 0.0 & 0.0 & 0.0 & 0.0 & 2.1 & 0.0 & 0.0 & 2.2 \\
\hline Pulp mills & 0.0 & 0.0 & 0.0 & 0.0 & 0.0 & 0.0 & 0.0 & 0.0 & 0.0 & 0.0 & 0.0 & 0.0 & 608.6 & 0.0 & 608.6 \\
\hline Sawmills & 5.5 & 0.0 & 8.6 & 47.1 & 67.2 & 5.8 & 0.0 & 0.0 & 0.0 & 1.2 & 0.0 & 0.0 & 28.6 & 25.4 & 189.2 \\
\hline Veneer \& plywood mfg & 0.0 & 0.0 & 0.0 & 0.0 & 0.0 & 0.0 & 0.0 & 0.0 & 0.0 & 0.0 & 51.5 & 0.0 & 0.0 & 0.0 & 51.5 \\
\hline Wood container/pallet mfg & 5.2 & 0.0 & 0.0 & 0.0 & 0.3 & 0.0 & 0.0 & 0.0 & 0.0 & 0.7 & 0.0 & 0.0 & 0.0 & 0.0 & 6.2 \\
\hline Wood preservation & 7.9 & 0.0 & 0.0 & 0.0 & 0.0 & 0.0 & 0.0 & 0.0 & 0.0 & 1.7 & 0.0 & 0.0 & 0.0 & 0.0 & 9.6 \\
\hline Wood windows/doors mfg & 0.0 & 0.0 & 1.1 & 0.0 & 0.0 & 0.0 & 0.0 & 0.0 & 0.0 & 0.0 & 0.0 & 0.9 & 0.0 & 0.0 & 2.0 \\
\hline Total & 70.4 & 8.6 & 18.1 & 79.3 & 99.1 & 21.4 & 3.8 & 25.0 & 4.8 & 31.7 & 70.7 & 87.5 & 692.4 & 34.7 & $1,247.7$ \\
\hline
\end{tabular}


Table 2(continued). Output (revenue) of agriculture and natural resource industries, Suwannee River Basin counties of Florida, 2002 data

\begin{tabular}{|c|c|c|c|c|c|c|c|c|c|c|c|c|c|c|c|}
\hline Industry Group & 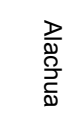 & 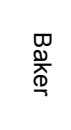 & 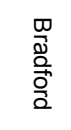 & $\begin{array}{l}\curvearrowright \\
\frac{\Omega}{\frac{O}{5}} \\
\frac{\bar{g}}{\bar{D}}\end{array}$ & $\begin{array}{l}\frac{D}{x} \\
\text { 离. }\end{array}$ & 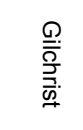 & 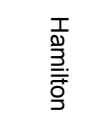 & 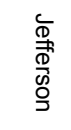 & 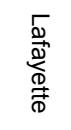 & $\stackrel{\Gamma}{\sum}$ & $\begin{array}{l}\frac{3}{0} \\
\frac{0}{0 .} \\
\stackrel{0}{0}\end{array}$ & 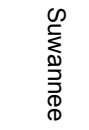 & $\begin{array}{l}\stackrel{-1}{\grave{2}} \\
\stackrel{0}{\varrho}\end{array}$ & ড়. & $\begin{array}{l}\text { Grand } \\
\text { Total }\end{array}$ \\
\hline Fruit \& Vegetable Farming \& Processing & $2<----$ & & & ------ & 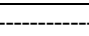 & $\$ M$ & $n$ & & & - & - & 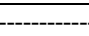 & ---.--> & & \\
\hline Fruit farming & 19.6 & 0.2 & 0.5 & 0.5 & 0.1 & 1.3 & 0.4 & 3.6 & 1.0 & 1.5 & 1.2 & 1.9 & 0.5 & 1.3 & 33.7 \\
\hline Vegetable \& melon farming & 17.1 & 7.7 & 0.7 & 0.2 & 2.8 & 7.5 & 1.8 & 1.0 & 1.5 & 19.4 & 2.1 & 31.6 & 0.0 & 1.1 & 94.5 \\
\hline Total & 36.7 & 7.9 & 1.2 & 0.7 & 2.9 & 8.8 & 2.2 & 4.6 & 2.5 & 20.9 & 3.3 & 33.5 & 0.5 & 2.4 & 128.2 \\
\hline \multicolumn{16}{|c|}{ Livestock \& Dairy Farming \& Animal Products Mfg } \\
\hline Animal production, except cattle \& poultry & 13.1 & 1.7 & 0.9 & 3.3 & 0.7 & 3.3 & 0.5 & 0.2 & 0.6 & 5.6 & 4.7 & 1.7 & 1.8 & 0.5 & 38.6 \\
\hline Animal, except poultry, slaughtering & 0.0 & 0.0 & 2.3 & 18.9 & 0.0 & 0.0 & 7.2 & 0.0 & 0.0 & 0.0 & 0.0 & 30.3 & 0.0 & 0.0 & 58.6 \\
\hline Cattle ranching \& farming & 14.4 & 2.8 & 9.6 & 7.5 & 7.0 & 34.1 & 4.9 & 14.4 & 21.4 & 29.1 & 5.5 & 23.7 & 1.7 & 4.0 & 180.2 \\
\hline Meat processed from carcasses & 0.0 & 0.0 & 0.0 & 0.0 & 0.0 & 0.0 & 0.0 & 0.0 & 0.0 & 0.0 & 100.2 & 0.0 & 0.0 & 0.0 & 100.2 \\
\hline Poultry \& egg production & 1.1 & 7.5 & 12.9 & 4.9 & 1.2 & 0.4 & 3.4 & 0.4 & 24.3 & 0.0 & 12.3 & 53.9 & 3.7 & 3.5 & 129.7 \\
\hline Poultry processing & 0.0 & 0.0 & 0.0 & 0.0 & 0.0 & 0.0 & 0.0 & 0.0 & 0.0 & 0.0 & 0.0 & 286.9 & 0.0 & 0.0 & 286.9 \\
\hline Rendering \&meat byproduct processing & 0.0 & 0.0 & 16.2 & 0.0 & 0.0 & 0.0 & 0.0 & 0.0 & 0.0 & 0.0 & 0.0 & 0.0 & 0.0 & 0.0 & 16.2 \\
\hline Total & 28.6 & 12.0 & 41.9 & 34.5 & 8.9 & 37.9 & 15.9 & 15.1 & 46.4 & 34.7 & 122.7 & 396.5 & 7.2 & 8.0 & 810.4 \\
\hline \multicolumn{16}{|l|}{ Mining } \\
\hline Drilling oil \& gas wells & 0.0 & 0.0 & 0.0 & 0.0 & 0.0 & 0.0 & 0.0 & 0.0 & 0.0 & 0.1 & 0.0 & 0.0 & 0.0 & 0.1 & 0.3 \\
\hline Gold/silver/other metal ore mining & 0.0 & 0.0 & 0.5 & 0.0 & 0.0 & 0.0 & 0.0 & 0.0 & 0.0 & 0.0 & 0.0 & 0.0 & 0.0 & 0.0 & 0.5 \\
\hline Oil \& gas extraction & 0.0 & 0.0 & 0.0 & 0.0 & 0.0 & 0.0 & 0.0 & 0.0 & 0.0 & 0.0 & 0.0 & 0.0 & 2.0 & 0.0 & 2.0 \\
\hline Other nonmetallic mineral mining & 0.0 & 0.0 & 0.2 & 0.0 & 0.0 & 0.0 & 0.0 & 0.0 & 0.0 & 0.0 & 0.0 & 0.0 & 0.6 & 0.0 & 0.9 \\
\hline Stone mining \& quarrying & 7.6 & 0.0 & 0.0 & 0.0 & 0.1 & 0.0 & 0.0 & 0.0 & 2.0 & 9.1 & 0.0 & 1.7 & 16.3 & 0.0 & 36.6 \\
\hline Support activities for oil \& gas & 0.3 & 0.0 & 0.1 & 0.0 & 0.0 & 0.0 & 0.0 & 0.0 & 0.0 & 0.0 & 0.0 & 0.0 & 0.0 & 0.0 & 0.5 \\
\hline Total & 7.9 & 0.0 & 0.9 & 0.0 & 0.1 & 0.0 & 0.0 & 0.0 & 2.0 & 9.2 & 0.0 & 1.7 & 18.9 & 0.1 & 40.8 \\
\hline \multicolumn{16}{|l|}{ Other Crop Farming } \\
\hline All other crop farming & 3.7 & 0.0 & 0.4 & 0.8 & 0.1 & 2.2 & 0.2 & 1.3 & 1.4 & 14.2 & 1.2 & 5.4 & 0.0 & 0.4 & 31.4 \\
\hline Cotton farming & 0.0 & 0.0 & 0.0 & 0.0 & 0.0 & 0.0 & 0.1 & 0.3 & 0.0 & 0.0 & 0.0 & 0.0 & 0.0 & 0.0 & 0.4 \\
\hline Grain farming & 0.0 & 0.0 & 0.0 & 0.0 & 0.0 & 0.1 & 0.3 & 1.4 & 0.1 & 0.1 & 0.6 & 0.4 & 0.0 & 0.0 & 3.0 \\
\hline Oilseed farming & 0.0 & 0.0 & 0.0 & 0.0 & 0.0 & 0.0 & 0.0 & 0.1 & 0.0 & 0.0 & 0.3 & 0.0 & 0.0 & 0.0 & 0.4 \\
\hline Tobacco farming & 2.3 & 0.1 & 0.0 & 0.6 & 0.0 & 0.4 & 1.2 & 0.5 & 1.8 & 0.8 & 2.5 & 7.6 & 0.0 & 0.2 & 17.9 \\
\hline Tree nut farming & 0.2 & 0.0 & 0.0 & 0.0 & 0.0 & 0.0 & 0.0 & 0.2 & 0.0 & 0.0 & 0.0 & 0.0 & 0.0 & 0.0 & 0.5 \\
\hline Total & 6.2 & 0.1 & 0.4 & 1.4 & 0.1 & 2.7 & 1.8 & 3.9 & 3.3 & 15.1 & 4.7 & 13.4 & 0.0 & 0.6 & 53.5 \\
\hline \multicolumn{16}{|l|}{ Other Food Product Manufacturing } \\
\hline Bread/bakery, except frozen, mfg & 3.6 & 0.0 & 0.0 & 0.0 & 0.0 & 0.0 & 0.6 & 0.0 & 0.0 & 0.0 & 0.0 & 0.0 & 0.3 & 0.0 & 4.4 \\
\hline Breweries & 3.1 & 0.0 & 0.0 & 0.0 & 0.0 & 0.0 & 0.0 & 0.0 & 0.0 & 0.0 & 0.0 & 0.0 & 0.0 & 0.0 & 3.1 \\
\hline Other animal food manufacturing & 0.0 & 0.0 & 0.0 & 17.7 & 0.0 & 0.0 & 0.0 & 0.0 & 0.0 & 0.0 & 0.0 & 4.7 & 0.0 & 7.6 & 29.9 \\
\hline Other snack food manufacturing & 0.0 & 0.0 & 0.0 & 0.0 & 0.0 & 0.0 & 0.0 & 0.0 & 0.0 & 0.0 & 0.0 & 0.0 & 12.9 & 0.0 & 12.9 \\
\hline Soft drink \& ice manufacturing & 4.2 & 0.0 & 0.0 & 0.0 & 0.0 & 0.0 & 1.5 & 0.0 & 0.0 & 0.0 & 0.0 & 0.0 & 0.0 & 0.0 & 5.7 \\
\hline Confectionery manufacturing & 1.2 & 0.0 & 0.0 & 0.0 & 0.0 & 0.0 & 0.0 & 0.0 & 0.0 & 0.0 & 0.0 & 0.0 & 0.0 & 0.0 & 1.2 \\
\hline Total & 12.0 & 0.0 & 0.0 & 17.7 & 0.0 & 0.0 & 2.1 & 0.0 & 0.0 & 0.0 & 0.0 & 4.7 & 13.2 & 7.6 & 57.2 \\
\hline Total Industry Output & 246.1 & 35.3 & 66.8 & 147.4 & 114.0 & 77.2 & 315.8 & 79.5 & 61.5 & 154.4 & 218.1 & 551.5 & 739.3 & 56.0 & $2,862.9$ \\
\hline
\end{tabular}

Source: IMPLAN data for Florida counties (2005) 
Table 3. Output impacts of agriculture and natural resource industries, Suwannee River Basin counties of Florida, 2002 data

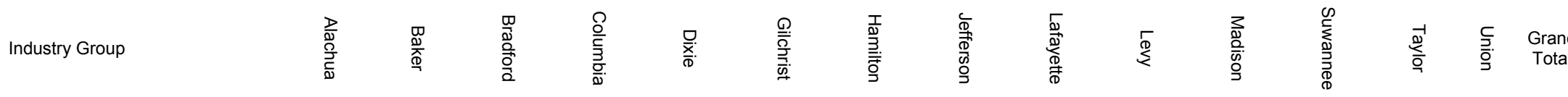

\begin{tabular}{|c|c|c|c|c|c|c|c|c|c|c|c|c|c|c|c|}
\hline Agricultural Inputs \& Services & & 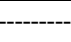 & 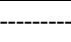 & (1) & ---- & ---- & \$Million & 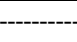 & 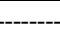 & - & ----- & -a--- & 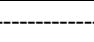 & $---->$ & \\
\hline Agriculture \& forestry activities & 25.9 & 0.0 & 0.2 & 4.9 & 0.0 & 1.3 & 0.3 & 1.2 & 0.1 & 22.4 & 12.4 & 3.5 & 3.5 & 0.2 & 76.0 \\
\hline Farm machinery \&equipment mfg & 0.0 & 0.0 & 0.0 & 0.0 & 0.0 & 0.0 & 0.0 & 0.0 & 0.0 & 0.0 & 0.0 & 1.2 & 0.0 & 0.0 & 1.2 \\
\hline Fertilizer, mixing only, mfg & 0.2 & 0.0 & 0.0 & 0.0 & 0.0 & 0.0 & 0.0 & 0.0 & 0.0 & 0.0 & 0.0 & 0.0 & 0.0 & 0.7 & 0.9 \\
\hline Food product machinery mfg & 0.0 & 0.0 & 0.0 & 0.0 & 0.0 & 0.0 & 0.0 & 0.5 & 0.0 & 0.0 & 0.0 & 0.0 & 0.0 & 0.0 & 0.5 \\
\hline Lawn/garden equipment mfg & 0.0 & 0.0 & 0.0 & 0.0 & 0.0 & 0.0 & 0.0 & 1.0 & 0.0 & 0.0 & 0.0 & 0.0 & 0.0 & 0.0 & 1.0 \\
\hline New farm housing units/additions & 7.8 & 0.5 & 0.6 & 1.8 & 1.3 & 0.3 & 0.2 & 0.4 & 0.0 & 1.2 & 0.2 & 0.8 & 0.7 & 0.2 & 15.7 \\
\hline Phosphatic fertilizer mfg & 0.0 & 0.0 & 0.0 & 0.0 & 0.0 & 0.0 & 374.4 & 0.0 & 0.0 & 0.0 & 0.0 & 0.0 & 0.0 & 0.0 & 374.4 \\
\hline Veterinary services & 31.9 & 1.5 & 0.8 & 2.2 & 0.3 & 0.2 & 0.7 & 1.8 & 0.2 & 3.2 & 0.3 & 3.0 & 0.6 & 0.0 & 46.8 \\
\hline Total & 65.8 & 2.0 & 1.5 & 8.9 & 1.6 & 1.8 & 375.6 & 4.9 & 0.3 & 26.8 & 12.9 & 8.5 & 4.8 & 1.1 & 516.4 \\
\hline \multicolumn{16}{|l|}{ Environmental Horticulture } \\
\hline Greenhouse/nursery production & 9.7 & 6.2 & 0.2 & 1.7 & 1.5 & 5.6 & 0.2 & 17.5 & 2.8 & 26.7 & 8.1 & 4.4 & 0.4 & 0.4 & 85.3 \\
\hline Landscape services ( $70 \%$ bldgs) & 41.9 & 0.6 & 2.9 & 6.5 & 0.2 & 0.5 & 0.3 & 1.6 & 0.1 & 2.0 & 1.1 & 3.1 & 1.9 & 1.0 & 63.7 \\
\hline Total & 51.5 & 6.8 & 3.1 & 8.2 & 1.7 & 6.1 & 0.6 & 19.1 & 2.9 & 28.7 & 9.2 & 7.5 & 2.3 & 1.4 & 149.0 \\
\hline \multicolumn{16}{|l|}{ Fishing and Wildlife } \\
\hline Fishing & 0.0 & 0.0 & 0.0 & 0.0 & 0.0 & 0.0 & 0.0 & 0.0 & 0.0 & 3.1 & 0.0 & 0.0 & 0.0 & 0.0 & 3.1 \\
\hline Wildlife (hunting \& trapping) & 0.0 & 0.0 & 0.0 & 0.0 & 0.0 & 0.0 & 0.0 & 16.7 & 0.0 & 0.0 & 0.0 & 0.0 & 0.0 & 0.0 & 16.7 \\
\hline Total & 0.0 & 0.0 & 0.0 & 0.0 & 0.0 & 0.0 & 0.0 & 16.7 & 0.0 & 3.1 & 0.0 & 0.0 & 0.0 & 0.0 & 19.8 \\
\hline \multicolumn{16}{|l|}{ Forestry, Wood \& Paper Product Mfg } \\
\hline Cut stock/resawing lumber/planting & 0.0 & 0.0 & 0.0 & 22.7 & 0.0 & 0.0 & 0.0 & 0.0 & 0.0 & 0.0 & 0.0 & 0.0 & 0.0 & 0.0 & 22.7 \\
\hline Engineered wood \& truss mfg & 19.1 & 0.0 & 0.0 & 0.7 & 0.0 & 2.0 & 0.0 & 0.0 & 0.7 & 0.0 & 0.0 & 0.0 & 0.0 & 0.0 & 22.5 \\
\hline Envelope manufacturing & 5.9 & 0.0 & 0.0 & 0.0 & 0.0 & 0.0 & 0.0 & 0.0 & 0.0 & 0.0 & 0.0 & 0.0 & 0.0 & 0.0 & 5.9 \\
\hline Forest nurseries/products/timber & 34.8 & 8.7 & 0.0 & 0.0 & 3.7 & 11.2 & 3.1 & 5.0 & 1.4 & 7.5 & 8.2 & 57.5 & 19.9 & 0.0 & 160.9 \\
\hline Logging & 7.2 & 2.8 & 9.4 & 16.3 & 29.6 & 5.6 & 2.2 & 29.0 & 4.2 & 38.8 & 17.3 & 64.9 & 44.2 & 9.5 & 280.8 \\
\hline Miscellaneous wood product mfg & 0.0 & 0.0 & 0.5 & 0.3 & 0.0 & 2.4 & 0.0 & 0.0 & 0.0 & 0.0 & 0.0 & 0.3 & 0.0 & 0.0 & 3.6 \\
\hline Other millwork, including flooring & 5.5 & 0.0 & 0.0 & 0.2 & 0.0 & 0.0 & 0.0 & 0.0 & 0.0 & 0.0 & 0.0 & 0.0 & 0.0 & 0.0 & 5.7 \\
\hline Prefabricated wood building mfg & 0.4 & 0.0 & 0.0 & 0.0 & 0.0 & 0.0 & 0.0 & 0.0 & 0.0 & 0.0 & 0.0 & 2.9 & 0.0 & 0.0 & 3.3 \\
\hline Pulp mills & 0.0 & 0.0 & 0.0 & 0.0 & 0.0 & 0.0 & 0.0 & 0.0 & 0.0 & 0.0 & 0.0 & 0.0 & 791.5 & 0.0 & 791.5 \\
\hline Sawmills & 6.1 & 0.0 & 15.1 & 82.6 & 108.7 & 9.7 & 0.0 & 0.0 & 0.0 & 1.3 & 0.0 & 0.0 & 29.5 & 38.3 & 291.2 \\
\hline Veneer \& plywood mfg & 0.0 & 0.0 & 0.0 & 0.0 & 0.0 & 0.0 & 0.0 & 0.0 & 0.0 & 0.0 & 81.2 & 0.0 & 0.0 & 0.0 & 81.2 \\
\hline Wood container/pallet mfg & 8.2 & 0.0 & 0.0 & 0.0 & 0.6 & 0.0 & 0.0 & 0.0 & 0.0 & 0.8 & 0.0 & 0.0 & 0.0 & 0.0 & 9.7 \\
\hline Wood preservation & 12.0 & 0.0 & 0.0 & 0.0 & 0.0 & 0.0 & 0.0 & 0.0 & 0.0 & 2.7 & 0.0 & 0.0 & 0.0 & 0.0 & 14.7 \\
\hline Wood windows/doors mfg & 0.0 & 0.0 & 1.4 & 0.0 & 0.0 & 0.0 & 0.0 & 0.0 & 0.0 & 0.0 & 0.0 & 1.0 & 0.0 & 0.0 & 2.4 \\
\hline Total & 99.1 & 11.4 & 26.4 & 122.8 & 142.6 & 30.9 & 5.2 & 34.0 & 6.3 & 51.1 & 106.7 & 126.7 & 885.0 & 47.7 & $1,696.0$ \\
\hline
\end{tabular}


Table 3 (continued). Output impacts of agriculture and natural resource industries, Suwannee River Basin counties of Florida, 2002 data

\begin{tabular}{|c|c|c|c|c|c|c|c|c|c|c|c|c|c|c|}
\hline Industry Group & 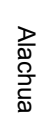 & 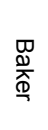 & 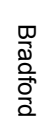 & $\frac{\frac{\delta}{\bar{C}}}{\frac{\mathrm{O}}{3}}$ & $\frac{\bar{D}}{\frac{\mathrm{x}}{\bar{D}}}$ & 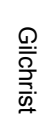 & 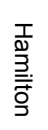 & $\begin{array}{l}\bar{\Phi} \\
\stackrel{\Phi}{*} \\
\frac{0}{0} \\
0 \\
0\end{array}$ & 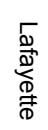 & $\stackrel{5}{<}$ & $\begin{array}{l}3 \\
\frac{3}{0} \\
\frac{2}{\bar{m}} \\
\frac{0}{3}\end{array}$ & 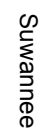 & 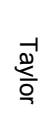 & $\frac{c}{\frac{5}{3}}$ \\
\hline
\end{tabular}

\begin{tabular}{|c|c|c|c|c|c|c|c|c|c|c|c|c|c|c|c|}
\hline Fruit \& Vegetable Farming \& Processing & & & & & 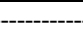 & 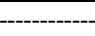 & - \$Million & & & & & 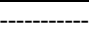 & 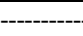 & -..-> & \\
\hline Fruit farming & 31.2 & 0.2 & 0.6 & 0.6 & 0.2 & 1.7 & 0.5 & 4.6 & 1.2 & 2.0 & 1.7 & 2.5 & 0.6 & 1.6 & 49.0 \\
\hline Vegetable \& melon farming & 25.1 & 10.6 & 0.8 & 0.2 & 3.7 & 9.9 & 2.2 & 1.2 & 1.9 & 29.9 & 2.8 & 44.7 & 0.0 & 1.3 & 134.3 \\
\hline Total & 56.3 & 10.8 & 1.3 & 0.8 & 3.8 & 11.6 & 2.7 & 5.8 & 3.1 & 31.9 & 4.5 & 47.2 & 0.6 & 2.9 & 183.3 \\
\hline \multicolumn{16}{|c|}{ Livestock \& Dairy Farming \& Animal Products Mfg } \\
\hline Animal production, except cattle \& poultry & 19.1 & 2.1 & 1.0 & 3.3 & 0.8 & 4.1 & 0.5 & 0.3 & 0.8 & 8.2 & 5.3 & 1.7 & 2.1 & 0.6 & 49.9 \\
\hline Animal, except poultry, slaughtering & 0.0 & 0.0 & 2.6 & 29.6 & 0.0 & 0.0 & 11.2 & 0.0 & 0.0 & 0.0 & 0.0 & 56.5 & 0.0 & 0.0 & 100.0 \\
\hline Cattle ranching \& farming & 23.9 & 3.9 & 12.9 & 7.5 & 9.2 & 48.2 & 5.2 & 21.0 & 29.4 & 50.4 & 5.5 & 28.4 & 2.2 & 5.4 & 253.2 \\
\hline Meat processed from carcasses & 0.0 & 0.0 & 0.0 & 0.0 & 0.0 & 0.0 & 0.0 & 0.0 & 0.0 & 0.0 & 132.0 & 0.0 & 0.0 & 0.0 & 132.0 \\
\hline Poultry \& egg production & 1.6 & 9.9 & 16.5 & 6.6 & 1.4 & 0.5 & 4.2 & 0.5 & 29.7 & 0.0 & 15.3 & 54.1 & 4.4 & 4.1 & 148.9 \\
\hline Poultry processing & 0.0 & 0.0 & 0.0 & 0.0 & 0.0 & 0.0 & 0.0 & 0.0 & 0.0 & 0.0 & 0.0 & 446.9 & 0.0 & 0.0 & 446.9 \\
\hline Rendering \& meat byproduct processing & 0.0 & 0.0 & 25.0 & 0.0 & 0.0 & 0.0 & 0.0 & 0.0 & 0.0 & 0.0 & 0.0 & 0.0 & 0.0 & 0.0 & 25.0 \\
\hline Total & 44.7 & 15.9 & 58.0 & 47.1 & 11.4 & 52.9 & 21.1 & 21.8 & 59.9 & 58.6 & 158.1 & 587.6 & 8.7 & 10.1 & $1,155.8$ \\
\hline \multicolumn{16}{|l|}{ Mining } \\
\hline Drilling oil \& gas wells & 0.0 & 0.0 & 0.0 & 0.0 & 0.0 & 0.0 & 0.0 & 0.0 & 0.0 & 0.2 & 0.0 & 0.0 & 0.0 & 0.1 & 0.3 \\
\hline Gold/silver/other metal ore mining & 0.0 & 0.0 & 0.8 & 0.0 & 0.0 & 0.0 & 0.0 & 0.0 & 0.0 & 0.0 & 0.0 & 0.0 & 0.0 & 0.0 & 0.8 \\
\hline Oil \& gas extraction & 0.0 & 0.0 & 0.0 & 0.0 & 0.0 & 0.0 & 0.0 & 0.0 & 0.0 & 0.0 & 0.0 & 0.0 & 2.5 & 0.0 & 2.5 \\
\hline Other nonmetallic mineral mining & 0.0 & 0.0 & 0.3 & 0.0 & 0.0 & 0.0 & 0.0 & 0.0 & 0.0 & 0.0 & 0.0 & 0.0 & 0.7 & 0.0 & 1.0 \\
\hline Stone mining \& quarrying & 14.2 & 0.0 & 0.0 & 0.0 & 0.1 & 0.0 & 0.0 & 0.0 & 2.4 & 13.1 & 0.0 & 2.4 & 20.4 & 0.0 & 52.6 \\
\hline Support activities for oil \& gas & 0.6 & 0.0 & 0.2 & 0.0 & 0.0 & 0.0 & 0.0 & 0.0 & 0.0 & 0.0 & 0.0 & 0.0 & 0.0 & 0.0 & 0.8 \\
\hline Total & 14.8 & 0.0 & 1.2 & 0.0 & 0.1 & 0.0 & 0.0 & 0.0 & 2.4 & 13.2 & 0.0 & 2.4 & 23.6 & 0.1 & 57.9 \\
\hline \multicolumn{16}{|l|}{ Other Crop Farming } \\
\hline All other crop farming & 5.2 & 0.0 & 0.4 & 0.8 & 0.1 & 2.3 & 0.2 & 1.3 & 1.4 & 18.1 & 1.3 & 5.5 & 0.0 & 0.4 & 37.0 \\
\hline Cotton farming & 0.0 & 0.0 & 0.0 & 0.0 & 0.0 & 0.0 & 0.1 & 0.4 & 0.0 & 0.0 & 0.0 & 0.0 & 0.0 & 0.0 & 0.5 \\
\hline Grain farming & 0.1 & 0.0 & 0.0 & 0.0 & 0.0 & 0.1 & 0.4 & 1.8 & 0.1 & 0.2 & 0.9 & 0.5 & 0.0 & 0.0 & 4.0 \\
\hline Oilseed farming & 0.0 & 0.0 & 0.0 & 0.0 & 0.0 & 0.0 & 0.0 & 0.2 & 0.0 & 0.0 & 0.5 & 0.0 & 0.0 & 0.0 & 0.7 \\
\hline Tobacco farming & 4.4 & 0.1 & 0.0 & 1.0 & 0.0 & 0.5 & 1.6 & 0.6 & 2.2 & 1.1 & 3.5 & 10.7 & 0.0 & 0.2 & 26.0 \\
\hline Tree nut farming & 0.4 & 0.0 & 0.0 & 0.0 & 0.0 & 0.0 & 0.0 & 0.3 & 0.0 & 0.0 & 0.0 & 0.0 & 0.0 & 0.0 & 0.7 \\
\hline Total & 10.0 & 0.1 & 0.4 & 1.8 & 0.1 & 2.8 & 2.3 & 4.7 & 3.8 & 19.4 & 6.2 & 16.7 & 0.0 & 0.6 & 68.9 \\
\hline \multicolumn{16}{|l|}{ Other Food Product Manufacturing } \\
\hline Bread/bakery, except frozen, mfg & 3.6 & 0.0 & 0.0 & 0.0 & 0.0 & 0.0 & 0.6 & 0.0 & 0.0 & 0.0 & 0.0 & 0.0 & 0.3 & 0.0 & 4.5 \\
\hline Breweries & 5.9 & 0.0 & 0.0 & 0.0 & 0.0 & 0.0 & 0.0 & 0.0 & 0.0 & 0.0 & 0.0 & 0.0 & 0.0 & 0.0 & 5.9 \\
\hline Other animal food manufacturing & 0.0 & 0.0 & 0.0 & 24.5 & 0.0 & 0.0 & 0.0 & 0.0 & 0.0 & 0.0 & 0.0 & 6.0 & 0.0 & 8.5 & 39.0 \\
\hline Other snack food manufacturing & 0.0 & 0.0 & 0.0 & 0.0 & 0.0 & 0.0 & 0.0 & 0.0 & 0.0 & 0.0 & 0.0 & 0.0 & 15.3 & 0.0 & 15.3 \\
\hline Soft drink \& ice manufacturing & 6.4 & 0.0 & 0.0 & 0.0 & 0.0 & 0.0 & 1.8 & 0.0 & 0.0 & 0.0 & 0.0 & 0.0 & 0.0 & 0.0 & 8.1 \\
\hline Confectionery manufacturing & 2.0 & 0.0 & 0.0 & 0.0 & 0.0 & 0.0 & 0.0 & 0.0 & 0.0 & 0.0 & 0.0 & 0.0 & 0.0 & 0.0 & 2.0 \\
\hline Total & 17.8 & 0.0 & 0.0 & 24.5 & 0.0 & 0.0 & 2.3 & 0.0 & 0.0 & 0.0 & 0.0 & 6.0 & 15.6 & 8.5 & 74.7 \\
\hline Total Output Impacts & 360.0 & 47.0 & 91.9 & 214.0 & 161.3 & 106.1 & 409.8 & 106.9 & 78.7 & 232.8 & 297.6 & 802.7 & 940.6 & 72.6 & $3,921.8$ \\
\hline
\end{tabular}

Source: IMPLAN data for Florida counties (2005) 
Table 4. Employment impacts of agriculture and natural resource industries, Suwannee River Basin counties of Florida, 2002 data

\begin{tabular}{|c|c|c|c|c|c|c|c|c|c|c|c|c|c|c|c|}
\hline Industry Group & $\begin{array}{l}\stackrel{\vec{D}}{\mathbb{N}} \\
\stackrel{\vec{D}}{\vec{Q}} \\
\vec{d}\end{array}$ & $\begin{array}{l}\text { W } \\
\stackrel{\mathbb{N}}{\hat{\widehat{D}}}\end{array}$ & 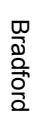 & $\begin{array}{l}\frac{\delta}{\frac{\delta}{2}} \\
\frac{3}{\frac{\partial}{\alpha}}\end{array}$ & $\frac{\bar{D}}{\mathrm{x}}$ & 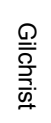 & 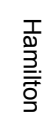 & 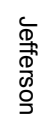 & 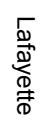 & $\stackrel{\Gamma}{\sum}$ & $\begin{array}{l}\frac{3}{3} \\
\frac{0}{0} \\
\frac{0}{0}\end{array}$ & 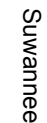 & 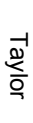 & 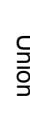 & $\begin{array}{c}\text { Grand } \\
\text { Total }\end{array}$ \\
\hline
\end{tabular}

\begin{tabular}{|c|c|c|c|c|c|c|c|c|c|c|c|c|c|c|c|}
\hline Agricultural Inputs \& Services & $<--$ & "--" & . & 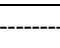 & - & ---- & obs ---- & 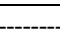 & 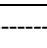 & -a-- & - & 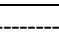 & $-\cdots$ & & \\
\hline Agriculture \& forestry activities & 320 & 0 & 7 & 65 & 0 & 49 & 13 & 33 & 5 & 333 & 356 & 38 & 74 & 10 & 1,303 \\
\hline Farm machinery \& equipment mfg & 0 & 0 & 0 & 0 & 0 & 0 & 0 & 0 & 0 & 0 & 0 & 7 & 0 & 0 & 7 \\
\hline Fertilizer, mixing only, mfg & 1 & 0 & 0 & 0 & 0 & 0 & 0 & 0 & 0 & 0 & 0 & 0 & 0 & 2 & 3 \\
\hline Food product machinery mfg & 0 & 0 & 0 & 0 & 0 & 0 & 0 & 5 & 0 & 0 & 0 & 0 & 0 & 0 & 5 \\
\hline Lawn/garden equipment $\mathrm{mfg}$ & 0 & 0 & 0 & 0 & 0 & 0 & 0 & 4 & 0 & 0 & 0 & 0 & 0 & 0 & 4 \\
\hline New farm housing units/additions & 46 & 3 & 4 & 10 & 7 & 2 & 1 & 2 & 0 & 7 & 1 & 6 & 4 & 1 & 95 \\
\hline Phosphatic fertilizer mfg & 0 & 0 & 0 & 0 & 0 & 0 & 1,305 & 0 & 0 & 0 & 0 & 0 & 0 & 0 & 1,305 \\
\hline Veterinary services & 569 & 33 & 18 & 39 & 8 & 5 & 13 & 37 & 5 & 61 & 5 & 63 & 9 & 0 & 865 \\
\hline Total & 937 & 36 & 28 & 113 & 15 & 56 & 1,333 & 81 & 10 & 401 & 362 & 114 & 87 & 14 & 3,588 \\
\hline \multicolumn{16}{|l|}{ Environmental Horticulture } \\
\hline Greenhouse/nursery production & 166 & 101 & 7 & 177 & 34 & 46 & 9 & 246 & 22 & 223 & 159 & 58 & 12 & 27 & 1,288 \\
\hline Landscape services ( $70 \%$ bldgs) & 1,069 & 19 & 66 & 164 & 4 & 14 & 9 & 43 & 2 & 48 & 42 & 86 & 63 & 27 & 1,656 \\
\hline Total & 1,235 & 120 & 73 & 340 & 39 & 60 & 18 & 289 & 24 & 271 & 201 & 144 & 74 & 55 & 2,944 \\
\hline
\end{tabular}

Fishing and Wildlife

Fishing

Wildlife (hunting \& trapping)

Total

$\begin{array}{llllllllllllll}0 & 0 & 0 & 0 & 0 & 0 & 0 & 0 & 0 & 57 & 0 & 0 & 0 & 0 \\ 0 & 0 & 0 & 0 & 0 & 0 & 0 & 233 & 0 & 0 & 0 & 0 & 0 & 0\end{array}$

\section{Forestry, Wood \& Paper Product Mfg}

Cut stock/resawing lumber/planting

Engineered wood \& truss mfg

Envelope manufacturing

Forest nurseries/products/timber

Logging

Miscellaneous wood product $\mathrm{mfg}$

Other millwork, including flooring

Prefabricated wood building $\mathrm{mfg}$

Pulp mills

Sawmills

Veneer \& plywood mfg

Wood container/pallet $\mathrm{mfg}$

Wood preservation

Wood windows/doors mfg

Total

$\begin{array}{rr}0 & 0 \\ 186 & 0 \\ 96 & 0 \\ 276 & 54 \\ 45 & 19 \\ 0 & 0 \\ 69 & 0 \\ 4 & 0 \\ 0 & 0 \\ 40 & 0 \\ 0 & 0 \\ 113 & 0 \\ 71 & 0 \\ 0 & 0 \\ 901 & 73\end{array}$

\begin{tabular}{rrrrrrrrrrrr}
0 & 0 & 0 & 0 & 0 & 0 & 0 & 57 & 0 & 0 & 0 & 0 \\
0 & 0 & 0 & 0 & 0 & 233 & 0 & 0 & 0 & 0 & 0 & 0 \\
233 \\
\hline
\end{tabular}

$73 \quad 186$

$\begin{array}{rr}0 & 136 \\ 0 & 7 \\ 0 & 0 \\ 0 & 0 \\ 60 & 85 \\ 4 & 3 \\ 0 & 3 \\ 0 & 0 \\ 0 & 0 \\ 110 & 656 \\ 0 & 0 \\ 0 & 0 \\ 0 & 0 \\ 12 & 0 \\ 186 & 889\end{array}$

$\begin{array}{rrrrr}0 & 0 & 0 & 0 & 0 \\ 0 & 24 & 0 & 0 & 9 \\ 0 & 0 & 0 & 0 & 0 \\ 25 & 99 & 29 & 41 & 10 \\ 158 & 31 & 14 & 0 & 26 \\ 0 & 23 & 0 & 195 & 0 \\ 0 & 0 & 0 & 0 & 0 \\ 0 & 0 & 0 & 0 & 0 \\ 0 & 0 & 0 & 0 & 0 \\ 719 & 70 & 0 & 0 & 0 \\ 0 & 0 & 0 & 0 & 0 \\ 8 & 0 & 0 & 0 & 0 \\ 0 & 0 & 0 & 0 & 0 \\ 0 & 0 & 0 & 0 & 0 \\ 909 & 247 & 43 & 237 & 45\end{array}$

$\begin{array}{rr}0 & 0 \\ 0 & 0 \\ 0 & 0 \\ 95 & 154 \\ 290 & 98 \\ 0 & 0 \\ 0 & 0 \\ 0 & 0 \\ 0 & 0 \\ 8 & 0 \\ 0 & 682 \\ 12 & 0 \\ 15 & 0 \\ 0 & 0 \\ 420 & 933\end{array}$

$\begin{array}{rrrr}0 & 0 & 0 & 136 \\ 0 & 0 & 0 & 226 \\ 0 & 0 & 0 & 96 \\ 337 & 186 & 0 & 1,307 \\ 308 & 182 & 62 & 1,376 \\ 3 & 0 & 0 & 229 \\ 0 & 0 & 0 & 72 \\ 27 & 0 & 0 & 31 \\ 0 & 3,130 & 0 & 3,130 \\ 0 & 182 & 275 & 2,060 \\ 0 & 0 & 0 & 682 \\ 0 & 0 & 0 & 133 \\ 0 & 0 & 0 & 86 \\ 9 & 0 & 0 & 21 \\ 685 & 3,679 & 337 & 9,585\end{array}$


Table 4 (continued). Employment impacts of agriculture and natural resource industries, Suwannee River Basin counties of Florida, 2002 data

\begin{tabular}{|c|c|c|c|c|c|c|c|c|c|c|c|c|c|c|c|}
\hline Industry Group & 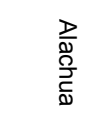 & $\begin{array}{l}\text { D. } \\
\stackrel{\mathbb{0}}{\mathbf{Q}}\end{array}$ & 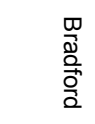 & $\begin{array}{l}\frac{\Omega}{0} \\
\frac{\delta}{5} \\
\frac{\sigma}{0}\end{array}$ & 离. & 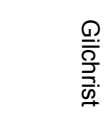 & $\begin{array}{l}\frac{\mathrm{T}}{\mathrm{D}} \\
\stackrel{3}{\overrightarrow{\underline{J}}} \\
\mathrm{O}\end{array}$ & 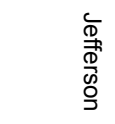 & 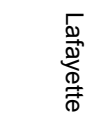 & $\stackrel{\infty}{<}$ & $\begin{array}{l}\frac{3}{0} \\
\frac{0}{0 .} \\
\frac{0}{0}\end{array}$ & 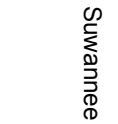 & 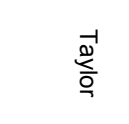 & $\begin{array}{l}\text { ํ. } \\
\text { ํ. }\end{array}$ & $\begin{array}{l}\text { Grand } \\
\text { Total }\end{array}$ \\
\hline Fruit \& Vegetable Farming \& Processing & $<-\ldots$ & 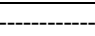 & 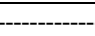 & 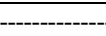 & 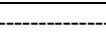 & 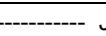 & bs - & & - & 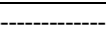 & 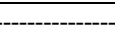 & -3 & ------> & & \\
\hline Fruit farming & 551 & 4 & 17 & 20 & 3 & 18 & 13 & 79 & 13 & 22 & 36 & 41 & 15 & 51 & 883 \\
\hline Vegetable \& melon farming & 300 & 125 & 16 & 8 & 54 & 62 & 47 & 10 & 10 & 201 & 37 & 371 & 0 & 42 & 1,283 \\
\hline Total & 851 & 128 & 33 & 29 & 58 & 80 & 60 & 89 & 23 & 223 & 73 & 412 & 15 & 93 & 2,165 \\
\hline \multicolumn{16}{|c|}{ Livestock \& Dairy Farming \& Animal Products Mfg } \\
\hline Animal production, except cattle \& poultry & 480 & 56 & 31 & 111 & 23 & 96 & 15 & 0 & 20 & 193 & 157 & 53 & 63 & 19 & 1,315 \\
\hline Animal, except poultry, slaughtering & 0 & 0 & 11 & 215 & 0 & 0 & 72 & 7 & 0 & 0 & 0 & 388 & 0 & 0 & 694 \\
\hline Cattle ranching \& farming & 274 & 42 & 147 & 83 & 100 & 484 & 57 & 227 & 296 & 523 & 59 & 301 & 26 & 65 & 2,683 \\
\hline Meat processed from carcasses & 0 & 0 & 0 & 0 & 0 & 0 & 0 & 0 & 0 & 0 & 913 & 0 & 0 & 0 & 913 \\
\hline Poultry \& egg production & 10 & 54 & 93 & 42 & 7 & 2 & 22 & 2 & 106 & 0 & 77 & 116 & 20 & 21 & 571 \\
\hline Poultry processing & 0 & 0 & 0 & 0 & 0 & 0 & 0 & 0 & 0 & 0 & 0 & 3,270 & 0 & 0 & 3,270 \\
\hline Rendering \& meat byproduct processing & 0 & 0 & 160 & 0 & 0 & 0 & 0 & 0 & 0 & 0 & 0 & 0 & 0 & 0 & 160 \\
\hline Total & 764 & 152 & 442 & 450 & 129 & 582 & 166 & 237 & 422 & 716 & 1,205 & 4,128 & 108 & 105 & 9,606 \\
\hline \multicolumn{16}{|l|}{ Mining } \\
\hline Drilling oil \& gas wells & 0 & 0 & 0 & 0 & 0 & 0 & 0 & 0 & 0 & 2 & 0 & 0 & 0 & 2 & 4 \\
\hline Gold/silver/other metal ore mining & 0 & 0 & 8 & 0 & 0 & 0 & 0 & 0 & 0 & 0 & 0 & 0 & 0 & 0 & 8 \\
\hline Oil \& gas extraction & 0 & 0 & 0 & 0 & 0 & 0 & 0 & 0 & 0 & 0 & 0 & 0 & 86 & 0 & 86 \\
\hline Other nonmetallic mineral mining & 0 & 0 & 3 & 0 & 0 & 0 & 0 & 0 & 0 & 0 & 0 & 0 & 5 & 0 & 9 \\
\hline Stone mining \& quarrying & 142 & 0 & 0 & 0 & 2 & 0 & 0 & 0 & 17 & 123 & 0 & 25 & 109 & 0 & 419 \\
\hline Support activities for oil \& gas & 13 & 0 & 4 & 0 & 0 & 0 & 0 & 0 & 0 & 0 & 0 & 0 & 0 & 0 & 17 \\
\hline Total & 155 & 0 & 16 & 0 & 2 & 0 & 0 & 0 & 17 & 126 & 0 & 25 & 200 & 2 & 543 \\
\hline \multicolumn{16}{|l|}{ Other Crop Farming } \\
\hline All other crop farming & 61 & 0 & 7 & 20 & 1 & 10 & 4 & 11 & 6 & 102 & 14 & 41 & 1 & 9 & 316 \\
\hline Cotton farming & 0 & 0 & 0 & 0 & 0 & 0 & 1 & 3 & 0 & 0 & 0 & 0 & 0 & 0 & 1 \\
\hline Grain farming & 1 & 0 & 0 & 1 & 0 & 1 & 16 & 34 & 1 & 2 & 23 & 9 & 0 & 0 & 88 \\
\hline Oilseed farming & 0 & 0 & 0 & 0 & 0 & 0 & 0 & 3 & 0 & 0 & 9 & 0 & 0 & 0 & 12 \\
\hline Tobacco farming & 87 & 2 & 0 & 67 & 0 & 4 & 68 & 10 & 18 & 10 & 79 & 143 & 0 & 15 & 503 \\
\hline Tree nut farming & 4 & 0 & 0 & 0 & 0 & 0 & 0 & 2 & 0 & 0 & 0 & 0 & 0 & 0 & 4 \\
\hline Total & 153 & 2 & 7 & 88 & 1 & 16 & 89 & 63 & 25 & 113 & 125 & 193 & 1 & 23 & 925 \\
\hline \multicolumn{16}{|l|}{ Other Food Product Manufacturing } \\
\hline Bread/bakery, except frozen, $\mathrm{mfg}$ & 56 & 0 & 0 & 0 & 0 & 0 & 6 & 0 & 0 & 0 & 0 & 0 & 4 & 0 & 66 \\
\hline Breweries & 60 & 0 & 0 & 0 & 0 & 0 & 0 & 0 & 0 & 0 & 0 & 0 & 0 & 0 & 60 \\
\hline Other animal food manufacturing & 0 & 0 & 0 & 120 & 0 & 0 & 0 & 0 & 0 & 0 & 0 & 24 & 0 & 28 & 173 \\
\hline Other snack food manufacturing & 0 & 0 & 0 & 0 & 0 & 0 & 0 & 0 & 0 & 0 & 0 & 0 & 77 & 0 & 77 \\
\hline Soft drink \& ice manufacturing & 46 & 0 & 0 & 0 & 0 & 0 & 9 & 0 & 0 & 0 & 0 & 0 & 0 & 0 & 55 \\
\hline Confectionery manufacturing & 19 & 0 & 0 & 0 & 0 & 0 & 0 & 0 & 0 & 0 & 0 & 0 & 0 & 0 & 19 \\
\hline Total & 181 & 0 & 0 & 121 & 0 & 0 & 16 & 0 & 0 & 0 & 0 & 25 & 81 & 28 & 450 \\
\hline Total Employment Impacts & 5,176 & 512 & 785 & 2,030 & 1,153 & 1,041 & 1,724 & 1,229 & 566 & 2,327 & 2,899 & 5,726 & 4,246 & 656 & 30,071 \\
\hline
\end{tabular}

Source: IMPLAN data for Florida counties (2005) 
Table 5. Value-added impacts of agriculture and natural resource industries, Suwannee River Basin counties of Florida, 2002 data

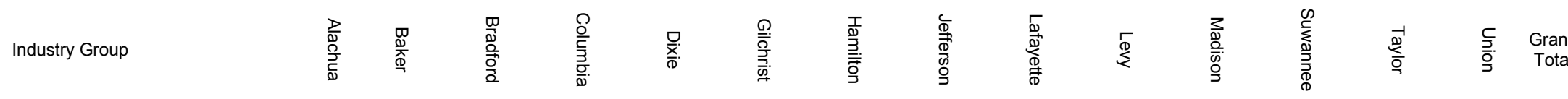

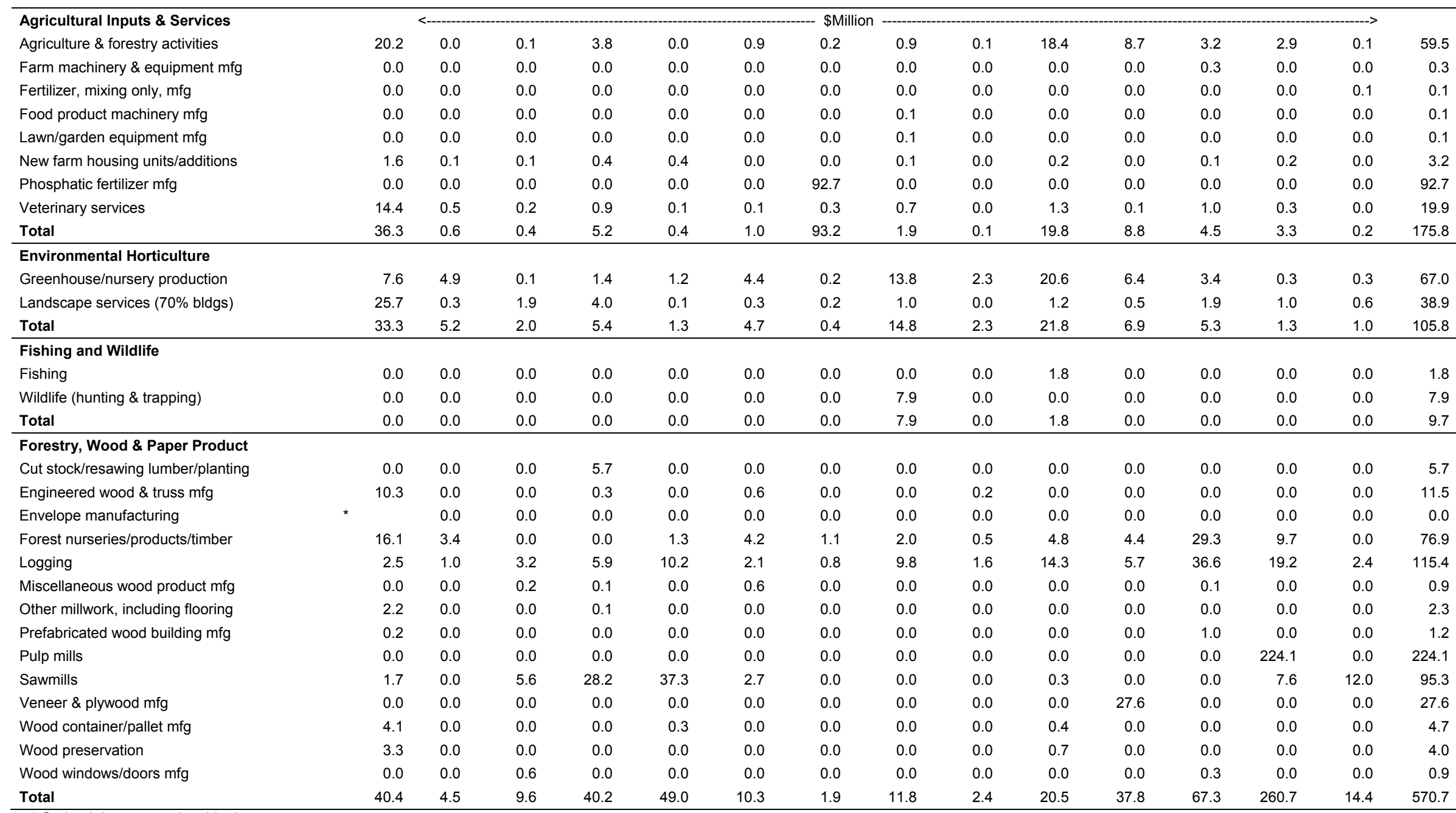

${ }^{*}$ Omitted due to questionable data. 
Table 5 (continued). Value-added impacts of agriculture and natural resource industries, Suwannee River Basin counties of Florida, 2002 data

\begin{tabular}{|c|c|c|c|c|c|c|c|c|c|c|c|c|c|c|c|}
\hline Industry Group & 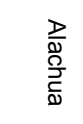 & 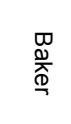 & 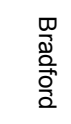 & 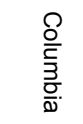 & 竞: & 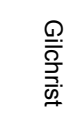 & 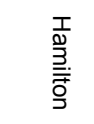 & 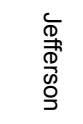 & 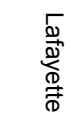 & $\stackrel{1}{<}$ & 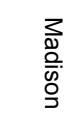 & 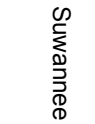 & 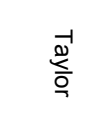 & 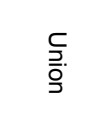 & $\begin{array}{c}\text { Grand } \\
\text { Total }\end{array}$ \\
\hline Fruit \& Vegetable Farming \& Processing & $<-\ldots$ & 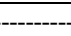 & & & 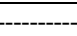 & 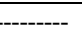 & illion - & & & - & & 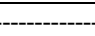 & 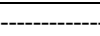 & & \\
\hline Fruit farming & 15.5 & 0.1 & 0.1 & 0.1 & 0.1 & 1.0 & 0.1 & 2.2 & 0.7 & 1.2 & 0.7 & 1.2 & 0.2 & 0.4 & 23.6 \\
\hline Vegetable \& melon farming & 14.8 & 6.2 & 0.4 & 0.1 & 2.1 & 5.8 & 1.2 & 0.7 & 1.1 & 17.7 & 1.6 & 25.8 & 0.0 & 0.7 & 78.3 \\
\hline Total & 30.3 & 6.3 & 0.5 & 0.2 & 2.1 & 6.8 & 1.4 & 2.9 & 1.8 & 18.9 & 2.4 & 27.0 & 0.2 & 1.1 & 101.9 \\
\hline \multicolumn{16}{|c|}{ Livestock \& Dairy Farming \& Animal Products Mfg } \\
\hline Animal production, except cattle \& poultry & 5.1 & 0.4 & 0.0 & 0.1 & 0.1 & 1.3 & 0.0 & 0.0 & 0.2 & 2.3 & 0.6 & 0.1 & 0.2 & 0.0 & 10.6 \\
\hline Animal, except poultry, slaughtering & 0.0 & 0.0 & 0.3 & 5.0 & 0.0 & 0.0 & 1.5 & 0.0 & 0.0 & 0.0 & 0.0 & 10.7 & 0.0 & 0.0 & 17.5 \\
\hline Cattle ranching \& farming & 5.7 & 0.6 & 1.0 & 0.0 & 0.8 & 10.4 & 0.2 & 3.0 & 5.1 & 12.7 & 0.2 & 2.7 & 0.2 & 0.4 & 42.9 \\
\hline Meat processed from carcasses & 0.0 & 0.0 & 0.0 & 0.0 & 0.0 & 0.0 & 0.0 & 0.0 & 0.0 & 0.0 & 29.1 & 0.0 & 0.0 & 0.0 & 29.1 \\
\hline Poultry \& egg production & 0.7 & 4.1 & 4.7 & 1.5 & 0.5 & 0.3 & 1.1 & 0.2 & 16.9 & 0.0 & 5.9 & 22.9 & 1.4 & 0.8 & 61.0 \\
\hline Poultry processing & 0.0 & 0.0 & 0.0 & 0.0 & 0.0 & 0.0 & 0.0 & 0.0 & 0.0 & 0.0 & 0.0 & 122.7 & 0.0 & 0.0 & 122.7 \\
\hline Rendering \& meat byproduct processing & 0.0 & 0.0 & 11.4 & 0.0 & 0.0 & 0.0 & 0.0 & 0.0 & 0.0 & 0.0 & 0.0 & 0.0 & 0.0 & 0.0 & 11.4 \\
\hline Total & 11.5 & 5.1 & 17.5 & 6.5 & 1.4 & 12.0 & 2.9 & 3.3 & 22.1 & 15.1 & 35.8 & 159.1 & 1.8 & 1.2 & 295.2 \\
\hline \multicolumn{16}{|l|}{ Mining } \\
\hline Drilling oil \& gas wells & 0.0 & 0.0 & 0.0 & 0.0 & 0.0 & 0.0 & 0.0 & 0.0 & 0.0 & 0.0 & 0.0 & 0.0 & 0.0 & 0.0 & 0.1 \\
\hline Gold/silver/other metal ore mining & 0.0 & 0.0 & 0.4 & 0.0 & 0.0 & 0.0 & 0.0 & 0.0 & 0.0 & 0.0 & 0.0 & 0.0 & 0.0 & 0.0 & 0.4 \\
\hline Oil \& gas extraction & 0.0 & 0.0 & 0.0 & 0.0 & 0.0 & 0.0 & 0.0 & 0.0 & 0.0 & 0.0 & 0.0 & 0.0 & 1.4 & 0.0 & 1.4 \\
\hline Other nonmetallic mineral mining & 0.0 & 0.0 & 0.2 & 0.0 & 0.0 & 0.0 & 0.0 & 0.0 & 0.0 & 0.0 & 0.0 & 0.0 & 0.4 & 0.0 & 0.6 \\
\hline Stone mining \& quarrying & 9.0 & 0.0 & 0.0 & 0.0 & 0.0 & 0.0 & 0.0 & 0.0 & 1.5 & 7.8 & 0.0 & 1.4 & 13.1 & 0.0 & 32.9 \\
\hline Support activities for oil \& gas & 0.4 & 0.0 & 0.1 & 0.0 & 0.0 & 0.0 & 0.0 & 0.0 & 0.0 & 0.0 & 0.0 & 0.0 & 0.0 & 0.0 & 0.5 \\
\hline Total & 9.4 & 0.0 & 0.7 & 0.0 & 0.0 & 0.0 & 0.0 & 0.0 & 1.5 & 7.9 & 0.0 & 1.4 & 14.9 & 0.0 & 35.9 \\
\hline \multicolumn{16}{|l|}{ Other Crop Farming } \\
\hline All other crop farming & 2.6 & 0.0 & 0.1 & 0.2 & 0.0 & 1.2 & 0.1 & 0.6 & 0.8 & 10.2 & 0.5 & 2.6 & 0.0 & 0.1 & 19.1 \\
\hline Cotton farming & 0.0 & 0.0 & 0.0 & 0.0 & 0.0 & 0.0 & 0.0 & 0.2 & 0.0 & 0.0 & 0.0 & 0.0 & 0.0 & 0.0 & 0.2 \\
\hline Grain farming & 0.0 & 0.0 & 0.0 & 0.0 & 0.0 & 0.0 & 0.2 & 0.9 & 0.1 & 0.1 & 0.5 & 0.3 & 0.0 & 0.0 & 2.1 \\
\hline Oilseed farming & 0.0 & 0.0 & 0.0 & 0.0 & 0.0 & 0.0 & 0.0 & 0.1 & 0.0 & 0.0 & 0.3 & 0.0 & 0.0 & 0.0 & 0.5 \\
\hline Tobacco farming & 2.9 & 0.1 & 0.0 & 0.7 & 0.0 & 0.3 & 1.0 & 0.4 & 1.5 & 0.7 & 2.3 & 6.9 & 0.0 & 0.2 & 16.9 \\
\hline Tree nut farming & 0.2 & 0.0 & 0.0 & 0.0 & 0.0 & 0.0 & 0.0 & 0.1 & 0.0 & 0.0 & 0.0 & 0.0 & 0.0 & 0.0 & 0.4 \\
\hline Total & 5.7 & 0.1 & 0.1 & 0.8 & 0.0 & 1.6 & 1.3 & 2.5 & 2.3 & 11.0 & 3.6 & 9.8 & 0.0 & 0.3 & 39.2 \\
\hline \multicolumn{16}{|l|}{ Other Food Product Manufacturing } \\
\hline Bread/bakery, except frozen, mfg & 1.4 & 0.0 & 0.0 & 0.0 & 0.0 & 0.0 & 0.3 & 0.0 & 0.0 & 0.0 & 0.0 & 0.0 & 0.1 & 0.0 & 1.8 \\
\hline Breweries & 3.8 & 0.0 & 0.0 & 0.0 & 0.0 & 0.0 & 0.0 & 0.0 & 0.0 & 0.0 & 0.0 & 0.0 & 0.0 & 0.0 & 3.8 \\
\hline Other animal food manufacturing & 0.0 & 0.0 & 0.0 & 5.5 & 0.0 & 0.0 & 0.0 & 0.0 & 0.0 & 0.0 & 0.0 & 1.2 & 0.0 & 1.3 & 8.0 \\
\hline Other snack food manufacturing & 0.0 & 0.0 & 0.0 & 0.0 & 0.0 & 0.0 & 0.0 & 0.0 & 0.0 & 0.0 & 0.0 & 0.0 & 6.3 & 0.0 & 6.3 \\
\hline Soft drink \& ice manufacturing & 2.1 & 0.0 & 0.0 & 0.0 & 0.0 & 0.0 & 0.4 & 0.0 & 0.0 & 0.0 & 0.0 & 0.0 & 0.0 & 0.0 & 2.5 \\
\hline Confectionery manufacturing & 1.0 & 0.0 & 0.0 & 0.0 & 0.0 & 0.0 & 0.0 & 0.0 & 0.0 & 0.0 & 0.0 & 0.0 & 0.0 & 0.0 & 1.0 \\
\hline Total & 8.2 & 0.0 & 0.0 & 5.5 & 0.0 & 0.0 & 0.7 & 0.0 & 0.0 & 0.0 & 0.0 & 1.2 & 6.4 & 1.3 & 23.3 \\
\hline Total Value-added Impacts & 175.1 & 21.7 & 30.8 & 64.0 & 54.2 & 36.2 & 101.8 & 44.9 & 32.6 & 116.9 & 95.3 & 275.5 & 288.8 & 19.6 & $1,357.5$ \\
\hline
\end{tabular}

Source: IMPLAN data for Florida counties (2005) 
Table 6. Labor income impacts of agriculture and natural resource industries, Suwannee River Basin counties of Florida, 2002 data

\begin{tabular}{|c|c|c|c|c|c|c|c|c|c|c|c|c|c|c|}
\hline Industry Group & 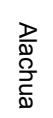 & 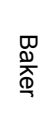 & $\begin{array}{l}\frac{w}{\mathbb{J}} \\
\frac{2}{0} \\
\frac{0}{2}\end{array}$ & $\begin{array}{l}\frac{\delta}{\frac{\delta}{5}} \\
\frac{3}{3}\end{array}$ & $\frac{\bar{D}}{\frac{D}{x}}$ & $\begin{array}{l}\frac{Q}{\underline{\underline{D}}} \\
\stackrel{\bar{D}}{\underline{\underline{D}}}\end{array}$ & 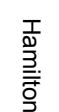 & 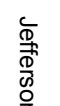 & 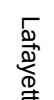 & $\stackrel{\text { D }}{\xi}$ & $\begin{array}{l}\frac{3}{0} \\
\frac{0}{0} \\
0\end{array}$ & 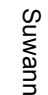 & & $\frac{c}{\mathrm{~J}}$ \\
\hline
\end{tabular}

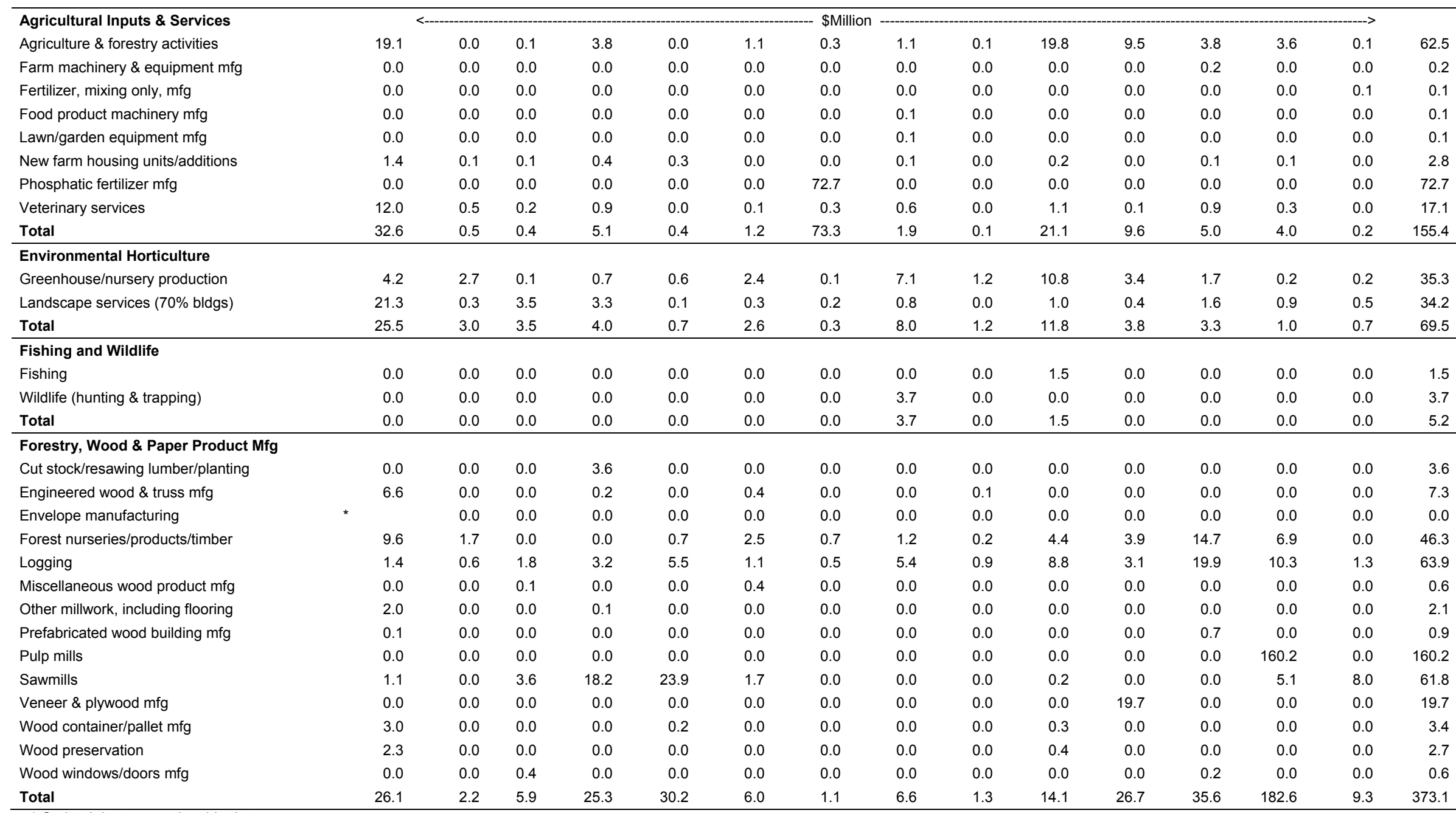

${ }^{*}$ Omitted due to questionable data. 
Table 6 (continued). Labor income impacts of agriculture and natural resource industries, Suwannee River Basin counties of Florida, 2002 data

\begin{tabular}{|c|c|c|c|c|c|c|c|c|c|c|c|c|c|c|c|}
\hline Industry Group & 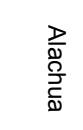 & $\begin{array}{l}\text { D. } \\
\stackrel{\mathbb{N}}{\mathbf{D}}\end{array}$ & 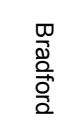 & 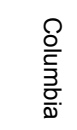 & 竞: & 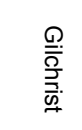 & 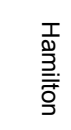 & 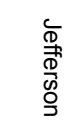 & 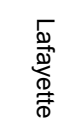 & $\stackrel{\Gamma}{<}$ & $\begin{array}{l}\frac{3}{30} \\
\frac{0}{0.0} \\
\frac{0}{3}\end{array}$ & 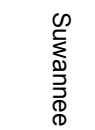 & 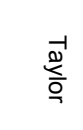 & $\begin{array}{l}\text { ㄷ․ } \\
\text { ㅁ. }\end{array}$ & $\begin{array}{l}\text { Grand } \\
\text { Total }\end{array}$ \\
\hline Fruit \& Vegetable Farming \& Processing & $<<-$ & & & & & ----- & llion & & & & & & & & \\
\hline Fruit farming & 9.4 & 0.0 & 0.1 & 0.1 & 0.0 & 0.6 & 0.1 & 1.2 & 0.4 & 0.7 & 0.5 & 0.6 & 0.1 & 0.2 & 13.9 \\
\hline Vegetable \&nd melon farming & 7.3 & 3.0 & 0.2 & 0.0 & 0.9 & 2.7 & 0.6 & 0.3 & 0.5 & 9.1 & 0.8 & 11.6 & 0.0 & 0.3 & 37.4 \\
\hline Total & 16.7 & 3.1 & 0.2 & 0.1 & 1.0 & 3.2 & 0.7 & 1.5 & 0.9 & 9.8 & 1.3 & 12.3 & 0.1 & 0.6 & 51.3 \\
\hline \multicolumn{16}{|c|}{ Livestock \& Dairy Farming \& Animal Products Mfg } \\
\hline Animal production, except cattle \& poultry & 3.0 & 0.3 & 0.0 & 0.0 & 0.1 & 0.8 & 0.0 & 0.0 & 0.1 & 1.4 & 0.4 & 0.1 & 0.1 & 0.0 & 6.4 \\
\hline Animal, except poultry, slaughtering & 0.0 & 0.0 & 0.2 & 3.5 & 0.0 & 0.0 & 1.1 & 0.0 & 0.0 & 0.0 & 0.0 & 6.6 & 0.0 & 0.0 & 11.5 \\
\hline Cattle ranching \& farming & 3.2 & 0.3 & 0.5 & 0.0 & 0.4 & 5.1 & 0.1 & 1.4 & 2.2 & 6.3 & 0.1 & 1.1 & 0.1 & 0.2 & 21.1 \\
\hline Meat processed from carcasses & 0.0 & 0.0 & 0.0 & 0.0 & 0.0 & 0.0 & 0.0 & 0.0 & 0.0 & 0.0 & 20.8 & 0.0 & 0.0 & 0.0 & 20.8 \\
\hline Poultry \& egg production & 0.4 & 2.0 & 2.0 & 0.7 & 0.2 & 0.1 & 0.5 & 0.1 & 6.2 & 0.0 & 2.4 & 6.5 & 0.5 & 0.3 & 21.9 \\
\hline Poultry processing & 0.0 & 0.0 & 0.0 & 0.0 & 0.0 & 0.0 & 0.0 & 0.0 & 0.0 & 0.0 & 0.0 & 84.4 & 0.0 & 0.0 & 84.4 \\
\hline Rendering \& meat byproduct processing & 0.0 & 0.0 & 6.1 & 0.0 & 0.0 & 0.0 & 0.0 & 0.0 & 0.0 & 0.0 & 0.0 & 0.0 & 0.0 & 0.0 & 6.1 \\
\hline Total & 6.6 & 2.6 & 8.8 & 4.3 & 0.7 & 6.1 & 1.8 & 1.5 & 8.5 & 7.8 & 23.6 & 98.7 & 0.7 & 0.6 & 172.2 \\
\hline \multicolumn{16}{|l|}{ Mining } \\
\hline Drilling oil \& gas wells & 0.0 & 0.0 & 0.0 & 0.0 & 0.0 & 0.0 & 0.0 & 0.0 & 0.0 & 0.0 & 0.0 & 0.0 & 0.0 & 0.0 & 0.1 \\
\hline Gold/silver/other metal ore mining & 0.0 & 0.0 & 0.3 & 0.0 & 0.0 & 0.0 & 0.0 & 0.0 & 0.0 & 0.0 & 0.0 & 0.0 & 0.0 & 0.0 & 0.3 \\
\hline Oil \& gas extraction & 0.0 & 0.0 & 0.0 & 0.0 & 0.0 & 0.0 & 0.0 & 0.0 & 0.0 & 0.0 & 0.0 & 0.0 & 0.6 & 0.0 & 0.6 \\
\hline Other nonmetallic mineral mining & 0.0 & 0.0 & 0.1 & 0.0 & 0.0 & 0.0 & 0.0 & 0.0 & 0.0 & 0.0 & 0.0 & 0.0 & 0.2 & 0.0 & 0.3 \\
\hline Stone mining \& quarrying & 5.4 & 0.0 & 0.0 & 0.0 & 0.0 & 0.0 & 0.0 & 0.0 & 0.9 & 4.4 & 0.0 & 0.8 & 7.3 & 0.0 & 18.8 \\
\hline Support activities for oil \& gas & 0.3 & 0.0 & 0.1 & 0.0 & 0.0 & 0.0 & 0.0 & 0.0 & 0.0 & 0.0 & 0.0 & 0.0 & 0.0 & 0.0 & 0.4 \\
\hline Total & 5.7 & 0.0 & 0.5 & 0.0 & 0.0 & 0.0 & 0.0 & 0.0 & 0.9 & 4.4 & 0.0 & 0.8 & 8.2 & 0.0 & 20.6 \\
\hline \multicolumn{16}{|l|}{ Other Crop Farming } \\
\hline All other crop farming & 1.3 & 0.0 & 0.0 & 0.1 & 0.0 & 0.4 & 0.0 & 0.2 & 0.2 & 4.0 & 0.2 & 0.8 & 0.0 & 0.0 & 7.4 \\
\hline Cotton farming & 0.0 & 0.0 & 0.0 & 0.0 & 0.0 & 0.0 & 0.0 & 0.1 & 0.0 & 0.0 & 0.0 & 0.0 & 0.0 & 0.0 & 0.1 \\
\hline Grain farming & 0.0 & 0.0 & 0.0 & 0.0 & 0.0 & 0.0 & 0.1 & 0.4 & 0.0 & 0.1 & 0.2 & 0.1 & 0.0 & 0.0 & 0.9 \\
\hline Oilseed farming & 0.0 & 0.0 & 0.0 & 0.0 & 0.0 & 0.0 & 0.0 & 0.1 & 0.0 & 0.0 & 0.2 & 0.0 & 0.0 & 0.0 & 0.2 \\
\hline Tobacco farming & 1.5 & 0.0 & 0.0 & 0.3 & 0.0 & 0.1 & 0.4 & 0.2 & 0.6 & 0.3 & 1.0 & 2.7 & 0.0 & 0.1 & 7.2 \\
\hline Tree nut farming & 0.1 & 0.0 & 0.0 & 0.0 & 0.0 & 0.0 & 0.0 & 0.1 & 0.0 & 0.0 & 0.0 & 0.0 & 0.0 & 0.0 & 0.2 \\
\hline Total & 2.9 & 0.0 & 0.0 & 0.4 & 0.0 & 0.6 & 0.6 & 1.0 & 0.8 & 4.4 & 1.6 & 3.7 & 0.0 & 0.1 & 16.1 \\
\hline \multicolumn{16}{|l|}{ Other Food Product Manufacturing } \\
\hline Bread/bakery, except frozen, mfg & 0.8 & 0.0 & 0.0 & 0.0 & 0.0 & 0.0 & 0.2 & 0.0 & 0.0 & 0.0 & 0.0 & 0.0 & 0.1 & 0.0 & 1.0 \\
\hline Breweries & 1.8 & 0.0 & 0.0 & 0.0 & 0.0 & 0.0 & 0.0 & 0.0 & 0.0 & 0.0 & 0.0 & 0.0 & 0.0 & 0.0 & 1.8 \\
\hline Other animal food manufacturing & 0.0 & 0.0 & 0.0 & 3.6 & 0.0 & 0.0 & 0.0 & 0.0 & 0.0 & 0.0 & 0.0 & 0.7 & 0.0 & 0.9 & 5.3 \\
\hline Other snack food manufacturing & 0.0 & 0.0 & 0.0 & 0.0 & 0.0 & 0.0 & 0.0 & 0.0 & 0.0 & 0.0 & 0.0 & 0.0 & 2.6 & 0.0 & 2.6 \\
\hline Soft drink \& ice manufacturing & 1.3 & 0.0 & 0.0 & 0.0 & 0.0 & 0.0 & 0.3 & 0.0 & 0.0 & 0.0 & 0.0 & 0.0 & 0.0 & 0.0 & 1.6 \\
\hline Confectionery manufacturing & 0.5 & 0.0 & 0.0 & 0.0 & 0.0 & 0.0 & 0.0 & 0.0 & 0.0 & 0.0 & 0.0 & 0.0 & 0.0 & 0.0 & 0.5 \\
\hline Total & 4.5 & 0.0 & 0.0 & 3.6 & 0.0 & 0.0 & 0.4 & 0.0 & 0.0 & 0.0 & 0.0 & 0.7 & 2.6 & 0.9 & 12.8 \\
\hline Total Labor Income Impacts & 120.6 & 11.4 & 19.5 & 42.8 & 33.0 & 19.8 & 78.1 & 24.2 & 13.6 & 74.9 & 66.6 & 159.9 & 199.3 & 12.5 & 876.2 \\
\hline
\end{tabular}

Source: IMPLAN data for Florida counties (2005) 
Table 7. Indirect business tax impacts of agriculture and natural resource industries, Suwannee River Basin counties of Florida, 2002 data

\begin{tabular}{|c|c|c|c|c|c|c|c|c|c|c|c|c|c|c|}
\hline Industry Group & 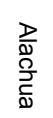 & 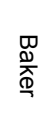 & $\begin{array}{l}\frac{w}{\mathbb{J}} \\
\frac{2}{0} \\
\frac{0}{2}\end{array}$ & $\begin{array}{l}\frac{\delta}{\frac{\delta}{5}} \\
\frac{3}{3}\end{array}$ & $\frac{\bar{D}}{\frac{D}{x}}$ & $\begin{array}{l}\frac{Q}{\underline{\underline{D}}} \\
\stackrel{\bar{D}}{\underline{\underline{D}}}\end{array}$ & 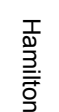 & 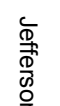 & 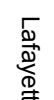 & $\stackrel{\text { D }}{\xi}$ & $\begin{array}{l}\frac{3}{0} \\
\frac{0}{0} \\
0\end{array}$ & 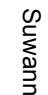 & & $\frac{c}{\mathrm{~J}}$ \\
\hline
\end{tabular}

\begin{tabular}{|c|c|c|c|c|c|c|c|c|c|c|c|c|c|c|c|}
\hline \multirow{2}{*}{$\begin{array}{l}\text { Agricultural Inputs \& Services } \\
\text { Agriculture \& forestry activities }\end{array}$} & \multicolumn{15}{|c|}{ <--> \$Million } \\
\hline & 0.9 & 0.0 & 0.0 & 0.2 & 0.0 & 0.0 & 0.0 & 0.0 & 0.0 & 0.6 & 0.3 & 0.0 & 0.0 & 0.0 & 2.0 \\
\hline Farm machinery \& equipment $\mathrm{mfg}$ & 0.0 & 0.0 & 0.0 & 0.0 & 0.0 & 0.0 & 0.0 & 0.0 & 0.0 & 0.0 & 0.0 & 0.0 & 0.0 & 0.0 & 0.0 \\
\hline Fertilizer, mixing only, mfg & 0.0 & 0.0 & 0.0 & 0.0 & 0.0 & 0.0 & 0.0 & 0.0 & 0.0 & 0.0 & 0.0 & 0.0 & 0.0 & 0.0 & 0.0 \\
\hline Food product machinery mfg & 0.0 & 0.0 & 0.0 & 0.0 & 0.0 & 0.0 & 0.0 & 0.0 & 0.0 & 0.0 & 0.0 & 0.0 & 0.0 & 0.0 & 0.0 \\
\hline Lawn/garden equipment mfg & 0.0 & 0.0 & 0.0 & 0.0 & 0.0 & 0.0 & 0.0 & 0.0 & 0.0 & 0.0 & 0.0 & 0.0 & 0.0 & 0.0 & 0.0 \\
\hline New farm housing units/additions & 0.0 & 0.0 & 0.0 & 0.0 & 0.0 & 0.0 & 0.0 & 0.0 & 0.0 & 0.0 & 0.0 & 0.0 & 0.0 & 0.0 & 0.1 \\
\hline Phosphatic fertilizer mfg & 0.0 & 0.0 & 0.0 & 0.0 & 0.0 & 0.0 & 0.0 & 0.0 & 0.0 & 0.0 & 0.0 & 0.0 & 0.0 & 0.0 & 0.0 \\
\hline Veterinary services & 1.0 & 0.0 & 0.0 & 0.1 & 0.0 & 0.0 & 0.0 & 0.1 & 0.0 & 0.1 & 0.0 & 0.1 & 0.0 & 0.0 & 1.4 \\
\hline Total & 1.9 & 0.0 & 0.0 & 0.2 & 0.0 & 0.0 & 5.1 & 0.1 & 0.0 & 0.7 & 0.3 & 0.1 & 0.1 & 0.0 & 8.6 \\
\hline \multicolumn{16}{|l|}{ Environmental Horticulture } \\
\hline Greenhouse/nursery production & 0.2 & 0.1 & 0.0 & 0.0 & 0.0 & 0.1 & 0.0 & 0.4 & 0.1 & 0.7 & 0.2 & 0.1 & 0.0 & 0.0 & 2.1 \\
\hline Landscape Services (70\% bldgs) & 1.0 & 0.0 & 1.2 & 0.2 & 0.0 & 0.0 & 0.0 & 0.0 & 0.0 & 0.1 & 0.0 & 0.1 & 0.0 & 0.0 & 2.6 \\
\hline Total & 1.3 & 0.1 & 1.2 & 0.2 & 0.0 & 0.1 & 0.0 & 0.5 & 0.1 & 0.8 & 0.2 & 0.2 & 0.0 & 0.0 & 4.7 \\
\hline \multicolumn{16}{|l|}{ Fishing and Wildlife } \\
\hline Fishing & 0.0 & 0.0 & 0.0 & 0.0 & 0.0 & 0.0 & 0.0 & 0.0 & 0.0 & 0.1 & 0.0 & 0.0 & 0.0 & 0.0 & 0.1 \\
\hline Wildlife (hunting \& trapping) & 0.0 & 0.0 & 0.0 & 0.0 & 0.0 & 0.0 & 0.0 & 0.9 & 0.0 & 0.0 & 0.0 & 0.0 & 0.0 & 0.0 & 0.9 \\
\hline Total & 0.0 & 0.0 & 0.0 & 0.0 & 0.0 & 0.0 & 0.0 & 0.9 & 0.0 & 0.1 & 0.0 & 0.0 & 0.0 & 0.0 & 1.0 \\
\hline \multicolumn{16}{|l|}{ Forestry, Wood \& Paper Product Mfg } \\
\hline Cut stock/resawing lumber/planting & 0.0 & 0.0 & 0.0 & 0.3 & 0.0 & 0.0 & 0.0 & 0.0 & 0.0 & 0.0 & 0.0 & 0.0 & 0.0 & 0.0 & 0.3 \\
\hline Engineered wood \& truss mfg & 0.4 & 0.0 & 0.0 & 0.0 & 0.0 & 0.0 & 0.0 & 0.0 & 0.0 & 0.0 & 0.0 & 0.0 & 0.0 & 0.0 & 0.4 \\
\hline Envelope manufacturing & 0.2 & 0.0 & 0.0 & 0.0 & 0.0 & 0.0 & 0.0 & 0.0 & 0.0 & 0.0 & 0.0 & 0.0 & 0.0 & 0.0 & 0.2 \\
\hline Forest nurseries/products/timber & 1.2 & 0.3 & 0.0 & 0.0 & 0.1 & 0.3 & 0.1 & 0.1 & 0.0 & 0.2 & 0.2 & 2.5 & 0.7 & 0.0 & 5.8 \\
\hline Logging & 0.1 & 0.0 & 0.1 & 0.2 & 0.3 & 0.1 & 0.0 & 0.4 & 0.1 & 0.6 & 0.2 & 1.6 & 0.6 & 0.1 & 4.2 \\
\hline Miscellaneous wood product mfg & 0.0 & 0.0 & 0.0 & 0.0 & 0.0 & 0.0 & 0.0 & 0.0 & 0.0 & 0.0 & 0.0 & 0.0 & 0.0 & 0.0 & 0.0 \\
\hline Other millwork, including flooring & 0.1 & 0.0 & 0.0 & 0.0 & 0.0 & 0.0 & 0.0 & 0.0 & 0.0 & 0.0 & 0.0 & 0.0 & 0.0 & 0.0 & 0.1 \\
\hline Prefabricated wood building mfg & 0.0 & 0.0 & 0.0 & 0.0 & 0.0 & 0.0 & 0.0 & 0.0 & 0.0 & 0.0 & 0.0 & 0.1 & 0.0 & 0.0 & 0.1 \\
\hline Pulp mills & 0.0 & 0.0 & 0.0 & 0.0 & 0.0 & 0.0 & 0.0 & 0.0 & 0.0 & 0.0 & 0.0 & 0.0 & 14.8 & 0.0 & 14.8 \\
\hline Sawmills & 0.0 & 0.0 & 0.2 & 1.5 & 1.2 & 0.1 & 0.0 & 0.0 & 0.0 & 0.0 & 0.0 & 0.0 & 0.2 & 0.3 & 3.5 \\
\hline Veneer \& plywood mfg & 0.0 & 0.0 & 0.0 & 0.0 & 0.0 & 0.0 & 0.0 & 0.0 & 0.0 & 0.0 & 1.1 & 0.0 & 0.0 & 0.0 & 1.1 \\
\hline Wood container/pallet mfg & 0.2 & 0.0 & 0.0 & 0.0 & 0.0 & 0.0 & 0.0 & 0.0 & 0.0 & 0.0 & 0.0 & 0.0 & 0.0 & 0.0 & 0.2 \\
\hline Wood preservation & 0.2 & 0.0 & 0.0 & 0.0 & 0.0 & 0.0 & 0.0 & 0.0 & 0.0 & 0.0 & 0.0 & 0.0 & 0.0 & 0.0 & 0.3 \\
\hline Wood windows/doors mfg & 0.0 & 0.0 & 0.0 & 0.0 & 0.0 & 0.0 & 0.0 & 0.0 & 0.0 & 0.0 & 0.0 & 0.0 & 0.0 & 0.0 & 0.0 \\
\hline Total & 2.3 & 0.3 & 0.3 & 2.0 & 1.6 & 0.5 & 0.1 & 0.5 & 0.1 & 0.9 & 1.5 & 4.2 & 16.2 & 0.4 & 31.0 \\
\hline
\end{tabular}


Table 7 (continued). Indirect business tax impacts of agriculture and natural resource industries, Suwannee River Basin counties of Florida, 2002 data

\begin{tabular}{|c|c|c|c|c|c|c|c|c|c|c|c|c|c|c|c|}
\hline Industry Group & 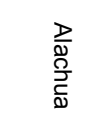 & 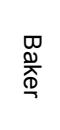 & 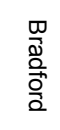 & $\frac{O}{\frac{O}{2}}$ & $\begin{array}{l}\frac{\bar{O}}{\mathrm{x}} \\
\overline{\mathbb{D}}\end{array}$ & 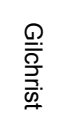 & 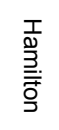 & 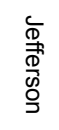 & 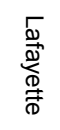 & $\stackrel{\Gamma}{\stackrel{D}{2}}$ & $\begin{array}{l}3 \\
\stackrel{3}{0} \\
\frac{0}{0} \\
\stackrel{0}{3}\end{array}$ & 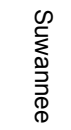 & 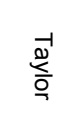 & ․ㅗ. & $\begin{array}{l}\text { Grand } \\
\text { Total }\end{array}$ \\
\hline Fruit \& Vegetable Farming \& Processing & $<<-+-$ & & & & & $-\mathbf{s}^{-1}$ & 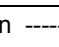 & & & & & & . & & \\
\hline Fruit farming & 1.0 & 0.0 & 0.0 & 0.0 & 0.0 & 0.1 & 0.0 & 0.1 & 0.0 & 0.1 & 0.0 & 0.1 & 0.0 & 0.0 & 1.5 \\
\hline Vegetable \& melon farming & 0.6 & 0.2 & 0.0 & 0.0 & 0.1 & 0.2 & 0.0 & 0.0 & 0.0 & 0.7 & 0.1 & 1.1 & 0.0 & 0.0 & 3.0 \\
\hline Total & 1.5 & 0.2 & 0.0 & 0.0 & 0.1 & 0.2 & 0.0 & 0.2 & 0.1 & 0.8 & 0.1 & 1.2 & 0.0 & 0.0 & 4.5 \\
\hline \multicolumn{16}{|c|}{ Livestock \& Dairy Farming \& Animal Products Mfg } \\
\hline Animal production, except cattle \& poultry & 0.5 & 0.0 & 0.0 & 0.0 & 0.0 & 0.1 & 0.0 & 0.0 & 0.0 & 0.2 & 0.1 & 0.0 & 0.1 & 0.0 & 1.0 \\
\hline Animal, except poultry, slaughtering & 0.0 & 0.0 & 0.0 & 0.4 & 0.0 & 0.0 & 0.1 & 0.0 & 0.0 & 0.0 & 0.0 & 1.1 & 0.0 & 0.0 & 1.7 \\
\hline Cattle ranching \& farming & 0.7 & 0.1 & 0.1 & 0.0 & 0.1 & 2.2 & 0.0 & 0.5 & 1.2 & 2.1 & 0.1 & 0.6 & 0.0 & 0.0 & 7.8 \\
\hline Meat processed from carcasses & 0.0 & 0.0 & 0.0 & 0.0 & 0.0 & 0.0 & 0.0 & 0.0 & 0.0 & 0.0 & 1.9 & 0.0 & 0.0 & 0.0 & 1.9 \\
\hline Poultry \& egg production & 0.0 & 0.1 & 0.2 & 0.1 & 0.0 & 0.0 & 0.0 & 0.0 & 0.5 & 0.0 & 0.2 & 0.4 & 0.0 & 0.0 & 1.6 \\
\hline Poultry processing & 0.0 & 0.0 & 0.0 & 0.0 & 0.0 & 0.0 & 0.0 & 0.0 & 0.0 & 0.0 & 0.0 & 7.3 & 0.0 & 0.0 & 7.3 \\
\hline Rendering \& meat byproduct processing & 0.0 & 0.0 & 0.4 & 0.0 & 0.0 & 0.0 & 0.0 & 0.0 & 0.0 & 0.0 & 0.0 & 0.0 & 0.0 & 0.0 & 0.4 \\
\hline Total & 1.2 & 0.3 & 0.7 & 0.5 & 0.2 & 2.4 & 0.2 & 0.5 & 1.8 & 2.3 & 2.2 & 9.4 & 0.1 & 0.1 & 21.7 \\
\hline \multicolumn{16}{|l|}{ Mining } \\
\hline Drilling oil \& gas wells & 0.0 & 0.0 & 0.0 & 0.0 & 0.0 & 0.0 & 0.0 & 0.0 & 0.0 & 0.0 & 0.0 & 0.0 & 0.0 & 0.0 & 0.0 \\
\hline Gold/silver/other metal ore mining & 0.0 & 0.0 & 0.0 & 0.0 & 0.0 & 0.0 & 0.0 & 0.0 & 0.0 & 0.0 & 0.0 & 0.0 & 0.0 & 0.0 & 0.0 \\
\hline Oil \& gas extraction & 0.0 & 0.0 & 0.0 & 0.0 & 0.0 & 0.0 & 0.0 & 0.0 & 0.0 & 0.0 & 0.0 & 0.0 & 0.2 & 0.0 & 0.2 \\
\hline Other nonmetallic mineral mining & 0.0 & 0.0 & 0.0 & 0.0 & 0.0 & 0.0 & 0.0 & 0.0 & 0.0 & 0.0 & 0.0 & 0.0 & 0.0 & 0.0 & 0.0 \\
\hline Stone mining \& quarrying & 0.5 & 0.0 & 0.0 & 0.0 & 0.0 & 0.0 & 0.0 & 0.0 & 0.1 & 0.4 & 0.0 & 0.1 & 0.7 & 0.0 & 1.8 \\
\hline Support activities for oil \& gas & 0.0 & 0.0 & 0.0 & 0.0 & 0.0 & 0.0 & 0.0 & 0.0 & 0.0 & 0.0 & 0.0 & 0.0 & 0.0 & 0.0 & 0.0 \\
\hline Total & 0.5 & 0.0 & 0.0 & 0.0 & 0.0 & 0.0 & 0.0 & 0.0 & 0.1 & 0.5 & 0.0 & 0.1 & 0.9 & 0.0 & 2.1 \\
\hline \multicolumn{16}{|l|}{ Other Crop Farming } \\
\hline All other crop farming & 0.2 & 0.0 & 0.0 & 0.0 & 0.0 & 0.1 & 0.0 & 0.0 & 0.0 & 0.6 & 0.0 & 0.2 & 0.0 & 0.0 & 1.1 \\
\hline Cotton farming & 0.0 & 0.0 & 0.0 & 0.0 & 0.0 & 0.0 & 0.0 & 0.0 & 0.0 & 0.0 & 0.0 & 0.0 & 0.0 & 0.0 & 0.0 \\
\hline Grain farming & 0.0 & 0.0 & 0.0 & 0.0 & 0.0 & 0.0 & 0.0 & 0.1 & 0.0 & 0.0 & 0.0 & 0.0 & 0.0 & 0.0 & 0.1 \\
\hline Oilseed farming & 0.0 & 0.0 & 0.0 & 0.0 & 0.0 & 0.0 & 0.0 & 0.0 & 0.0 & 0.0 & 0.0 & 0.0 & 0.0 & 0.0 & 0.0 \\
\hline Tobacco farming & 0.2 & 0.0 & 0.0 & 0.0 & 0.0 & 0.0 & 0.1 & 0.0 & 0.1 & 0.0 & 0.1 & 0.4 & 0.0 & 0.0 & 1.0 \\
\hline Tree nut farming & 0.0 & 0.0 & 0.0 & 0.0 & 0.0 & 0.0 & 0.0 & 0.0 & 0.0 & 0.0 & 0.0 & 0.0 & 0.0 & 0.0 & 0.0 \\
\hline Total & 0.3 & 0.0 & 0.0 & 0.1 & 0.0 & 0.1 & 0.1 & 0.1 & 0.1 & 0.7 & 0.2 & 0.6 & 0.0 & 0.0 & 2.3 \\
\hline \multicolumn{16}{|l|}{ Other Food Product Manufacturing } \\
\hline Bread/bakery, except frozen, mfg & 0.0 & 0.0 & 0.0 & 0.0 & 0.0 & 0.0 & 0.0 & 0.0 & 0.0 & 0.0 & 0.0 & 0.0 & 0.0 & 0.0 & 0.0 \\
\hline Breweries & 0.7 & 0.0 & 0.0 & 0.0 & 0.0 & 0.0 & 0.0 & 0.0 & 0.0 & 0.0 & 0.0 & 0.0 & 0.0 & 0.0 & 0.7 \\
\hline Other animal food manufacturing & 0.0 & 0.0 & 0.0 & 0.5 & 0.0 & 0.0 & 0.0 & 0.0 & 0.0 & 0.0 & 0.0 & 0.1 & 0.0 & 0.1 & 0.7 \\
\hline Other snack food manufacturing & 0.0 & 0.0 & 0.0 & 0.0 & 0.0 & 0.0 & 0.0 & 0.0 & 0.0 & 0.0 & 0.0 & 0.0 & 0.2 & 0.0 & 0.2 \\
\hline Soft drink \& ice manufacturing & 0.1 & 0.0 & 0.0 & 0.0 & 0.0 & 0.0 & 0.0 & 0.0 & 0.0 & 0.0 & 0.0 & 0.0 & 0.0 & 0.0 & 0.1 \\
\hline Confectionery manufacturing & 0.0 & 0.0 & 0.0 & 0.0 & 0.0 & 0.0 & 0.0 & 0.0 & 0.0 & 0.0 & 0.0 & 0.0 & 0.0 & 0.0 & 0.0 \\
\hline Total & 0.9 & 0.0 & 0.0 & 0.5 & 0.0 & 0.0 & 0.0 & 0.0 & 0.0 & 0.0 & 0.0 & 0.1 & 0.2 & 0.1 & 1.8 \\
\hline Total Indirect Business Tax Impacts & 10.0 & 0.9 & 2.2 & 3.5 & 1.8 & 3.4 & 5.6 & 2.8 & 2.3 & 6.6 & 4.6 & 15.8 & 17.6 & 0.6 & 77.8 \\
\hline
\end{tabular}

Source: IMPLAN data for Florida counties (2005) 